\title{
The transfer matrix in four-dimensional CDT
}

\author{
J. Ambjørn, ${ }^{a}$ J. Gizbert-Studnicki, ${ }^{b}$ A.T. Görlich ${ }^{a, b}$ and J. Jurkiewicz ${ }^{b}$ \\ ${ }^{a}$ The Niels Bohr Institute, Copenhagen University, \\ Blegdamsvej 17, DK-2100 Copenhagen Ø, Denmark \\ ${ }^{b}$ Institute of Physics, Jagellonian University, \\ Reymonta 4, PL 30-059 Krakow, Poland \\ E-mail: ambjorn@nbi.dk, jakub.gizbert-studnicki@uj.edu.pl, \\ goerlich@nbi.dk, jurkiewicz@th.if.uj.edu.pl
}

AbStract: The Causal Dynamical Triangulation model of quantum gravity (CDT) has a transfer matrix, relating spatial geometries at adjacent (discrete lattice) times. The transfer matrix uniquely determines the theory. We show that the measurements of the scale factor of the (CDT) universe are well described by an effective transfer matrix where the matrix elements are labeled only by the scale factor. Using computer simulations we determine the effective transfer matrix elements and show how they relate to an effective minisuperspace action at all scales.

KeYwORDS: Models of Quantum Gravity, Random Systems

ARXIV EPRINT: 1205.3791 


\section{Contents}

1 Introduction 1

2 CDT and the CDT transfer matrix 2

3 How to perform the computer simulations $\quad 7$

4 The effective action at large three-volumes 11

4.1 The kinetic term 11

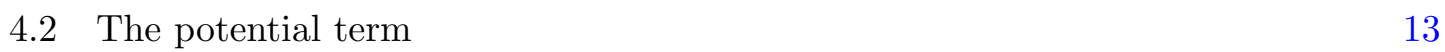

$\begin{array}{ll}4.3 \text { A global effective action fit } & 14\end{array}$

$\begin{array}{lll}4.4 \text { Miscellaneous } & 15\end{array}$

5 The transfer matrix for small three-volumes $\quad 17$

$\begin{array}{lll}5.1 \text { Eigenvectors analysis } & 17\end{array}$

$\begin{array}{ll}5.2 \text { The "full-CDT" approximation } & 19\end{array}$

$6 \quad$ The effective action for small three-volumes 19

$\begin{array}{lll}7 & \text { Discussion and conclusions } & 23\end{array}$

\section{Introduction}

Minisuperspace models of the universe provide us with simple quantum mechanical models of fluctuations of the scale factor of the universe. In the simplest models one assumes spatial homogeneity and isotropy. Classically this implies that we can write

$$
d s^{2}=-d t^{2}+a^{2}(t) d \Omega^{2}
$$

where $d \Omega^{2}$ is the line element of a homogeneous and isotropic three-dimensional space. Assuming space is compact it is $S^{3}$. Under these assumptions the dynamical variable is the scale factor $a(t)$ of the universe and the quantum field theory of the universe is reduced to quantum mechanics of a single variable $a(t)$.

The 4D Causal Dynamical Triangulation model (CDT) of quantum gravity is by construction a (regularized) quantum field theory model which is compatible with spatial homogeneity and isotropy (for reviews see [1-3]). It uses the path integral formulation and assumes there exists a foliation in (proper) time. When the average over all geometries of this kind is performed one indeed finds that the average geometry, i.e. what one naively would think is closest to a "classical geometry", can be described by a line element of type (1.1). Even more, it turns out that to a good approximation the scale factor $a(t)$, 
measured as the average of the third root of the spatial volume at (proper) time $t$ is well described by the simplest minisuperspace action. Contrary to the standard use of minisuperspace, where one postulates the reduction to geometries described by a metric like (1.1) and then only quantizes the single degree of freedom $a(t)$, the CDT discussion of $a(t)$ is exact (to the extent that CDT describes quantum gravity). The $a(t)$ entering in the CDT discussion is obtained by including all geometries in the path integral. It is natural to ask the following question: how well does the simplest minisuperspace action describe the CDT data generated by Monte Carlo simulations. The tool we will use when trying to answer this question is the transfer matrix.

The time foliation present in CDT provides us with a transfer matrix. The geometries considered in the (regularized) path integral are piecewise linear geometries constructed in such a way that at discretized times $t_{n}$ the spatial slices are triangulations of $S^{3}$. The transfer matrix relates a given spatial (piecewise linear) geometry at time $t_{n}$ to a given spatial geometry at time $t_{n+1}$. In ordinary (Euclidean) lattice field theory reflection positiveness of the transfer matrix ensures a unitary time evolution. The CDT transfer matrix has a similar property $[3-5] .{ }^{1}$ In this article we will analyze an "effective" transfer matrix of CDT to be defined below.

\section{CDT and the CDT transfer matrix}

The use of piecewise linear geometries was introduced in the context of general relativity by Regge [23] as a natural tool to work with a discretized version of the Hilbert-Einstein action, but without the use of coordinates. The idea was to approximate a given smooth geometry by a continuous, piecewise linear geometry. This piecewise linear geometry is uniquely defined by a triangulation where the length of the links are given. The observation by Regge was that the standard Einstein-Hilbert action in $D$ dimensions,

$$
S_{H E}[g]=\frac{1}{16 \pi G} \int d^{D} x \sqrt{-g}(R-2 \Lambda),
$$

for such piecewise linear geometries has a geometric interpretation as a sum over deficit angles of the $D$-2-dimensional sub-simplices in the triangulation. While the idea of Regge was to approach a given classically smooth geometry by a sequence of suitable piecewise linear triangulations, a different use of piecewise linear geometries was made in the formalism of Dynamical Triangulations (DT) [24-29]. Although we have no mathematical rigorous definition of the path integral over geometries, it is natural, in analogy with the path integral in ordinary quantum mechanics, to assume that the summation over geometries will involve not only smooth geometries but all continuous geometries. A subclass of these geometries is the piecewise linear geometries and a further subclass is the piecewise linear geometries defined by triangulations obtained by gluing together equilateral $D$-simplices such that they

\footnotetext{
${ }^{1}$ The idea of a time foliation and the fact that there exists a related unitary time evolution are features which CDT shares with Hořava-Lifshitz gravity [6-9]. However, no spatial higher derivative terms are explicitly added to the action, like in Hořava-Lifshitz gravity, and it is possible that fixed points of the lattice theory can be identified with the non-trivial UV fixed points conjectured in the asymptotic safety scenario suggested by Weinberg [10] and investigated in [11-22].
} 
form a manifold of fixed topology. In DT the assumption is that this set of geometries is in a suitable sense dense in the set of continuous geometries when we take the link length $a$ to zero, and in this way the link length $a$ will act as an ultraviolet cutoff, just like in ordinary lattice field theory. Further, the natural signature of space-time in the DT formulation is Euclidean. We will assume this is the case in the rest of this article. That this approach works in principle, i.e. that it is able to reproduce a continuum quantum field theory which is diffeomorphism invariant, is well documented for $D=2$. Two-dimensional Euclidean quantum gravity coupled to a conformal field theories with $c \leq 1$ can be solved analytically both in the continuum [30-32] and using the formalism of DT [33, 34] and agreement is found.

In higher dimensions DT was studied numerically, using Monte Carlo simulations both in three dimensions [35-39] and four dimensions [40, 41]. However, no convincing continuum limit has been obtained so far in higher dimensions [42-44], and this was one of the motivations for changing the class of triangulations used in the path integral in CDT. ${ }^{2}$ In the CDT formalism one sums over geometries with a (proper) time foliation. In principle one starts out with space-times with a Lorentzian signature (contrary to the situation in DT) and the foliation is in proper (Lorentzian) time. However, each piecewise linear geometry used in the CDT path integral allows a rotation to Euclidean proper time. The set of Euclidean geometries we obtain in this way is a subset of the DT Euclidean geometries and this restriction seemingly cures some of the higher dimensional DT diseases, while in two dimensions the relation between the restricted theory and the full DT theory has been worked out in detail: one obtains the CDT theory from the DT theory by integrating out all baby universes (which results in a non-analytic mapping between the coupling constants of the two theories), and (somewhat surprisingly) one can restore the DT theory from the CDT theory by the inverse mapping $[45,46]$. Using four-simplices (which is the case having our attention in this article) as building blocks one can, for suitable choices of bare coupling constants, observe a four-dimensional (Euclidean) universe [47, 48]. For these choices of coupling constants the shape of the universe is consistent with an interpretation as an (Euclidean) de Sitter space, at least as long as one looks at the scale factor [49, 50]. This is the region of coupling constants which will have our interest (for other choices of the coupling constants one obtains more degenerate configurations [51-53]).

An interesting feature of the CDT model, rotated to Euclidean signature, is that it possesses a transfer matrix [3-5]. At (discrete) time $t_{n}$ we have a spatial hypersurface with a spatial geometry characterized by a three-dimensional triangulation $T_{3}\left(t_{n}\right)$. At time $t_{n+1}$ we have spatial geometry defined by another three-dimensional triangulation $T_{3}\left(t_{n+1}\right)$. We assume for simplicity that the topology ${ }^{3}$ of the spatial triangulations is that of $S^{3}$. In CDT we sum over all four-dimensional triangulations of the "slab" between $t_{n}$ and $t_{n+1}$ compatible with the topology $S^{3} \times[0,1]$ and such that each four-simplex which "fills" the slab has subsimplices which are (sub)simplices of $T_{3}\left(t_{n}\right)$ as well as $T_{3}\left(t_{n+1}\right)$. This leads to 4 types of four-simplices in the slab: type $(4,1),(2,3),(3,2)$ and $(1,4)$, where the numbers

\footnotetext{
${ }^{2}$ It has recently been suggested that there might be a continuum limit of DT which belongs to the same universality class as the CDT theory described below [58].

${ }^{3} S^{3}$ is chosen for simplicity. We could have chosen any spatial topology. The important point in the assumption is that the topology is not allowed to change from one spatial hypersurface to the next.
} 
denote the number of vertices in $T_{3}\left(t_{n}\right)$ and $T_{3}\left(t_{n+1}\right)$ respectively. The number $N^{(4,1)}\left(t_{n}\right)$ of $(4,1)$ simplices in the slab is equal to the number $N_{3}\left(t_{n}\right)$ of three-simplices in $T_{3}\left(t_{n}\right)$, and similarly the number of $(1,4)$ simplices in the slab is equal to the number $N_{3}\left(t_{n+1}\right)$ of three-simplices in $T_{3}\left(t_{n+1}\right)$. The transfer matrix $\mathcal{M}$, i.e. the amplitude between the $T_{3}\left(t_{n}\right)$ and $T_{3}\left(t_{n+1}\right)$, is now given as the sum over all such triangulation,

$$
\left\langle T_{3}\left(t_{n+1}\right)|\mathcal{M}| T_{3}\left(t_{n}\right)\right\rangle=\sum_{T_{4}} \frac{1}{C_{T_{4}}} \mathrm{e}^{-S\left[T_{4}\right]},
$$

where the summation is over all four-dimensional triangulations of a slab, with boundary triangulations $T_{3}\left(t_{n}\right)$ and $T_{3}\left(t_{n+1}\right), C_{T_{4}}$ is the order of the automorphism group of the triangulation $T_{4}$, and where $S\left[T_{4}\right]$ is the Regge action of the four-dimensional triangulation of the slab.

The transfer matrix is defined on the vector space spanned by the set $\mathcal{T}_{3}$ of threedimensional triangulations. This space is infinite dimensional and has the natural scalar product

$$
\left\langle T \mid T^{\prime}\right\rangle=\frac{1}{C_{T}} \delta_{T, T^{\prime}}, \quad \sum_{T}|T\rangle C_{T}\langle T|=\hat{I}, \quad T, T^{\prime} \in \mathcal{T}_{3}
$$

where $C_{T}$ is the order of the automorphism group of the triangulation $T$.

The transition amplitude for a three-dimensional triangulation $T$ to develop into a three-dimensional triangulation $T^{\prime}$ after $t_{\text {tot }}+1$ (integer) time steps is

$$
\left\langle T^{\prime}\left|\mathcal{M}^{t_{\mathrm{tot}}+1}\right| T\right\rangle=\sum_{\left\{T_{i}\right\}}\left\langle T^{\prime}|\mathcal{M}| T_{t_{\mathrm{tot}}}\right\rangle C_{T_{t_{\mathrm{tot}}}}\left\langle T_{t_{\mathrm{tot}}}|\mathcal{M}| T_{t_{\mathrm{tot}}-1}\right\rangle \cdots C_{T_{1}}\left\langle T_{1}|\mathcal{M}| T\right\rangle .
$$

We will define the partition function corresponding to $t_{\text {tot }}$ time steps as path integral with periodic boundary conditions after $t_{\text {tot }}$ time-steps:

$$
\mathcal{Z}_{t_{\mathrm{tot}}}=\operatorname{tr} \mathcal{M}^{t_{\mathrm{tot}}}=\sum_{T} C_{T}\left\langle T\left|\mathcal{M}^{t_{\mathrm{tot}}}\right| T\right\rangle .
$$

This is the partition function we have used in our computer simulations. The measurements performed so far, using Monte Carlo simulations, have been concentrated on the measurement of the scale factor, or more conveniently the three-volume $n_{t_{i}} \equiv N_{3}\left(t_{i}\right)$ at the spatial slice at time $t_{i}$, as well as the correlation between the three-volumes at time $t_{i}$ and time $t_{j}$. These "observables" can be expressed using the transfer matrix $\mathcal{M}$. The probability of measuring the spatial volume $n_{t_{i}}$ at time $t_{i}$ is given by

$$
P^{t_{\mathrm{tot}}}\left(n_{t_{i}}\right)=\frac{1}{\mathcal{Z}_{\mathrm{ttot}_{\mathrm{tot}}}} \sum_{T \in \mathcal{T}_{3}\left(n_{t_{i}}\right)} C_{T}\left\langle T\left|\mathcal{M}^{t_{\mathrm{tot}}}\right| T\right\rangle=\frac{\operatorname{tr} \tilde{\rho}\left(n_{t_{i}}\right) \mathcal{M}^{t_{\mathrm{tot}}}}{\operatorname{tr} \mathcal{M}^{t_{\mathrm{tot}}}} .
$$

In (2.6) $\mathcal{T}_{3}\left(n_{t_{i}}\right)$ denotes the subset of three-dimensional triangulations where the number of three-simplices is $n_{t_{i}}$ and $\tilde{\rho}\left(n_{t_{i}}\right)$ the projection operator on the subspace spanned by these triangulations:

$$
\tilde{\rho}(n)=\sum_{T \in \mathcal{T}_{3}(n)}|T\rangle C_{T}\langle T|, \quad \tilde{\rho}(n)^{2}=\tilde{\rho}(n) .
$$


Similarly the correlator between $n_{t_{1}}$ and $n_{t_{2}}$, separated by $\Delta t=t_{2}-t_{1}$ is given by

$$
\begin{aligned}
P^{t_{\text {tot }}}\left(n_{t_{1}}, n_{t_{2}}\right) & =\frac{1}{\mathcal{Z}_{t_{\text {tot }}}} \sum_{\substack{T_{1} \in \mathcal{T}_{3}\left(n_{t_{1}}\right) \\
T_{2} \in \mathcal{T}_{3}\left(n_{t_{2}}\right)}} C_{T_{1}}\left\langle T_{1}\left|\mathcal{M}^{t_{\text {tot }}-\Delta t}\right| T_{2}\right\rangle C_{T_{2}}\left\langle T_{2}\left|\mathcal{M}^{\Delta t}\right| T_{1}\right\rangle \\
& =\frac{\operatorname{tr} \tilde{\rho}\left(n_{t_{1}}\right) \mathcal{M}^{t_{\text {tot }}-\Delta t} \tilde{\rho}\left(n_{t_{2}}\right) \mathcal{M}^{\Delta t}}{\operatorname{tr} \mathcal{M}^{t_{\text {tot }}}},
\end{aligned}
$$

and this expression can clearly be generalized to multi-correlators.

As mentioned in the Introduction it was possible to explain the observed distributions $P^{t_{\text {tot }}}\left(n_{t_{1}}\right)$ and $P^{t_{\text {tot }}}\left(n_{t_{1}}, n_{t_{2}}\right)$ using a simple minisuperspace model. How does the concept of a minisuperspace labeled by states $n_{t_{i}}, i=1, \ldots, t_{\text {tot }}$ relate to the transfer matrix $\mathcal{M}$ which is defined on the much larger space spanned by the vectors $\mathcal{T}$ ? Let us define the "effective" transfer matrix $M$ by

$$
|n\rangle\langle n|M| m\rangle\left\langle m\left|=\sum_{\substack{T_{n} \in \mathcal{T}_{3}(n) \\ T_{m} \in \mathcal{T}_{3}(m)}}\right| T_{n}\right\rangle C_{T_{n}}\left\langle T_{n}|\mathcal{M}| T_{m}\right\rangle C_{T_{m}}\left\langle T_{m}\right| .
$$

$\langle n|M| m\rangle$ represents the average of the matrix elements $\left\langle T_{n}|\mathcal{M}| T_{m}\right\rangle$ like

$$
\langle n|M| m\rangle=\langle\mathcal{M}\rangle_{n, m}:=\frac{1}{\mathcal{N}_{n} \mathcal{N}_{m}} \sum_{\substack{T_{n} \in \mathcal{T}_{3}(n) \\ T_{m} \in \mathcal{T}_{3}(m)}} \sqrt{C_{T_{n}} C_{T_{m}}}\left\langle T_{n}|\mathcal{M}| T_{m}\right\rangle,
$$

where $\mathcal{N}_{n}$ denotes the cardinality of $\mathcal{T}_{n} . \mathcal{N}_{n}$ grows exponentially with $n$.

In (2.9) and (2.10) it is misleading to think of the "state" $|n\rangle$ as the (suitably) normalized sum of the $\mathcal{N}_{n}$ vectors $\left|T_{n}\right\rangle$ (although by doing so one would of course obtain the correct expectation value $\langle\mathcal{M}\rangle_{n, m}$ using such vectors). Such a vector would again be a single vector located in the $\mathcal{N}_{n}$-dimensional space spanned by the $\left|T_{n}\right\rangle$ 's. It is more appropriate to think of the "state" associated with $n$ as arising from a uniform probability distribution of states $\left|T_{n}\right\rangle$ and in this way to think of $\tilde{\rho}(n)$ as the associated density operator. However, once we have reduced our consideration to the matrix $\langle n|M| m\rangle$ we are of course free to find eigenvectors for this matrix and expand them in the abstract basis $|n\rangle$, and we will indeed do that.

The statement that we can use the matrix $\langle n|M| m\rangle$ as an effective transfer matrix is the statement that the standard deviation of the $\mathcal{N}_{n} \mathcal{N}_{m}$ numbers $\left\langle T_{n}|\mathcal{M}| T_{m}\right\rangle$ is sufficiently small. In fact the difference between $\operatorname{tr} \mathcal{M}^{2}$ and $\operatorname{tr} M^{2}$ can exactly be expressed as a sum over deviations squared for each $n, m$ :

$$
\operatorname{tr} \mathcal{M}^{2}-\operatorname{tr} M^{2}=\sum_{n, m} \sum_{\substack{T_{n} \in \mathcal{T}_{3}(n) \\ T_{m} \in \mathcal{T}_{3}(m)}}\left(\sqrt{C_{T_{n}} C_{T_{m}}}\left\langle T_{n}|\mathcal{M}| T_{m}\right\rangle-\langle\mathcal{M}\rangle_{n, m}\right)^{2}
$$

In the following we will assume that we can work with an effective transfer matrix $\langle n|M| m\rangle$. Eq. (2.9) is an attempt to define this effective transfer matrix from first principles and in principle one can check by computer simulations if it is a good approximation. We will here take the pragmatic attitude to assume there exists such an effective transfer 
matrix and use it to analyze the computer generated data. The consistency of this analysis is indirectly evidence that an object like $\langle n|M| m\rangle$ provides a good approximation of our data. Thus we will use the "effective" version of (2.6)-(2.8):

$$
\begin{aligned}
\rho(n) & =|n\rangle\langle n|, \\
P^{t_{\mathrm{tot}}}\left(n_{t_{i}}\right) & =\frac{\operatorname{tr} \rho\left(n_{t_{i}}\right) M^{t_{\mathrm{tot}}}}{\operatorname{tr} M^{t_{\mathrm{tot}}}} . \\
P^{t_{\mathrm{tot}}}\left(n_{t_{1}}, n_{t_{2}}\right) & =\frac{\operatorname{tr} \rho\left(n_{t_{1}}\right) M^{t_{\mathrm{tot}}-\Delta t} \rho\left(n_{t_{2}}\right) M^{\Delta t}}{\operatorname{tr} M^{t_{\mathrm{tot}}}},
\end{aligned}
$$

where $\rho(n)$ should be distinguished from $\tilde{\rho}(n)$.

In particular we can measure the matrix elements $\langle n|M| m\rangle$ up to a normalization by considering $t_{\text {tot }}=2$. We have:

$$
P^{(2)}\left(n_{1}, n_{2}\right)=\frac{\left\langle n_{1}|M| n_{2}\right\rangle\left\langle n_{2}|M| n_{1}\right\rangle}{\operatorname{tr} M^{2}}
$$

This method requires a major change in our general computer program which assumes $t_{\text {tot }} \geq 3$. We updated it but we were not completely convinced that the new version is stable although it gave exactly the same results as the method we finally used. For $t_{\text {tot }}=3,4$ we get:

$$
\begin{aligned}
& P^{(3)}\left(n_{1}, n_{2}\right)=\frac{\left\langle n_{1}|M| n_{2}\right\rangle\left\langle n_{2}\left|M^{2}\right| n_{1}\right\rangle}{\operatorname{tr} M^{3}} \\
& P^{(4)}\left(n_{1}, n_{3}\right)=\frac{\left\langle n_{1}\left|M^{2}\right| n_{3}\right\rangle\left\langle n_{3}\left|M^{2}\right| n_{1}\right\rangle}{\operatorname{tr} M^{4}}
\end{aligned}
$$

From the measurements of $P^{(3)}\left(n_{1}, n_{2}\right)$ and $P^{(4)}\left(n_{1}, n_{3}\right)$ we can determine the matrix elements $\langle n|M| m\rangle$ up to a normalization:

$$
\langle n|M| m\rangle=C \frac{P^{(3)}\left(n_{1}=n, n_{2}=m\right)}{\sqrt{P^{(4)}\left(n_{1}=n, n_{3}=m\right)}}
$$

There is nothing magic about the above choice. One could have chosen $t_{\text {tot }}=4$ and $t_{\text {tot }}=6$ and formed the combinations

$$
\begin{aligned}
& P^{(4)}\left(n_{1}, n_{2}\right)=\frac{\left\langle n_{1}|M| n_{2}\right\rangle\left\langle n_{2}\left|M^{3}\right| n_{1}\right\rangle}{\operatorname{tr} M^{4}} \\
& P^{(6)}\left(n_{1}, n_{4}\right)=\frac{\left\langle n_{1}\left|M^{3}\right| n_{4}\right\rangle\left\langle n_{4}\left|M^{3}\right| n_{1}\right\rangle}{\operatorname{tr} M^{6}}
\end{aligned}
$$

from which one can again extract $\langle n|M| m\rangle$ like in (2.18). We have indeed checked that measurements of $P^{(4)}\left(n_{1}, n_{2}\right)$ and $P^{(6)}\left(n_{1}, n_{4}\right)$ lead to the same $M$ matrix as extracted from measurements of $P^{(3)}\left(n_{1}, n_{2}\right)$ and $P^{(4)}\left(n_{1}, n_{3}\right)$, up to a normalization.

In earlier work we have shown $[49,50,54]$ that the following minisuperspace action: ${ }^{4}$

$$
S\left[\left\{n_{t}\right\}\right]=\sum_{t} \frac{1}{\Gamma}\left[\frac{\left(n_{t+1}-n_{t}\right)^{2}}{n_{t}+n_{t+1}}+\mu\left(\frac{n_{t}+n_{t+1}}{2}\right)^{1 / 3}-\lambda \frac{n_{t}+n_{t+1}}{2}\right],
$$

${ }^{4}$ In fact we used slightly different form of the potential terms: $\mu\left(\frac{n_{t}+n_{t+1}}{2}\right)^{1 / 3}-\lambda \frac{n_{t}+n_{t+1}}{2} \Rightarrow \mu n_{t}^{1 / 3}-\lambda n_{t}$. This parametrization was more convenient to extract the parameters of the action from the measured covariance matrix of volume fluctuations. In this article we implement a modified form (2.21) which better fits our data. 
describes well the measured $\left\langle n_{t}\right\rangle$ and the fluctuations $\left\langle n_{t} n_{t^{\prime}}\right\rangle-\left\langle n_{t}\right\rangle\left\langle n_{t^{\prime}}\right\rangle$ in the bulk where $n_{t}$ is large. The effective action (2.21) suggests that the effective transfer matrix

$$
\langle n|M| m\rangle \propto e^{-\frac{1}{\Gamma}\left[\frac{(n-m)^{2}}{n+m}+\mu\left(\frac{n+m}{2}\right)^{1 / 3}-\lambda \frac{n+m}{2}\right]}
$$

is a good approximation in the bulk. We will in the following try to determine the transfer matrix from the data, also in the range where $n_{t}$ is not necessarily large and we will try to improve the expression (2.22).

\section{How to perform the computer simulations}

The simplest version of the discretized CDT theory has three parameters, two related to the cosmological constant and the gravitational constant, and an additional parameter which controls the asymmetry between the edge lengths in the spatial and time directions. This latter parameter seems not to be a genuine coupling constant since it just labels the different length assignment of spatial and time-like links. The action used is still the EinsteinHilbert action (as formulated by Regge for piecewise linear geometries), adjusted for this asymmetry. However, because we study the theory in a truly non-perturbative region of coupling constant space the effective action is determined by a competition between the classical action used and a contribution coming from the measure term. The contribution from the measure term is "entropic" in nature: it counts the number of configurations with the same action and is thus independent of the other parameters. Effectively this promotes the asymmetry parameter to a genuine coupling constant (see [3] for a detailed discussion).

In the numerical simulations the topology of the manifold is assumed to be $S^{3} \times S^{1}$ with periodic boundary conditions in the (Euclidean) time, as mentioned above. The foursimplices used to construct the simplicial manifolds of CDT are characterized by their position in spatial and time directions. As also mentioned above we have four types of four-simplices: $(4,1)$-simplices, with four vertices at time $t$ and one vertex at $t+1,(3,2)$ simplices with three simplices at $t$ and two at $t+1$ and the "time-reversed" $(1,4)$-simplices and $(2,3)$-simplices. All simplices of a particular type are identical.

The discretized (Regge) Einstein-Hilbert action becomes extremely simple because we are essentially only using the two kinds of building blocks to construct the four-dimensional triangulation $T[3-5]$ :

$$
S_{R}[T]=-\left(\kappa_{0}+6 \Delta\right) N_{0}+\kappa_{4}\left(N^{(4,1)}+N^{(3,2)}\right)+\Delta\left(2 N^{(4,1)}+N^{(3,2)}\right)
$$

where $N_{0}$ is the total number of vertices in the triangulation, $N^{(4,1)}$ the total number of type $(4,1)$ plus $(1,4)$ simplices and $N^{(3,2)}$ the total number of simplices of type $(3,2)$ plus $(2,3)$. $\kappa_{0}, \kappa_{4}$ and $\Delta$ are the (bare) dimensionless coupling constants obtained by the discretization of the continuous action (2.1). $\kappa_{0}$ is proportional to the inverse bare gravitational constant, $\kappa_{4}$ related to the cosmological constant while $\Delta$ is related to the asymmetry between the spatial and time-like links. $\Delta=0$ corresponds to spatial and time-like links having the same length. An additional geometric parameter is the length $t_{\text {tot }}$ of the periodic time axis. 
The partition function

$$
\mathcal{Z}=\sum_{T \in \mathcal{T}_{4}} e^{-S_{R}[T]}
$$

has a critical value $\kappa_{4}^{\text {crit }}\left(\kappa_{0}, \Delta\right)$, depending on $\kappa_{0}$ and $\Delta$, such that $\mathcal{Z}$ is divergent for $\kappa_{4}<\kappa_{4}^{c r i t}$. The existence of this critical value is reflecting the fact that the number of triangulations with a fixed number of four-simplices $N_{4}$ grows exponentially with $N_{4}$. In principle we want to fine tune $\kappa_{4}$ to this critical value since we really want a limit where $N_{4} \rightarrow \infty$. In practice the simulations have so far been carried out by keeping $N_{4}$ (or $N^{(4,1)}$ ) fixed. In this way we have been trading the coupling constant $\kappa_{4}$ with $N_{4}$ and the partition function $\mathcal{Z}\left(N_{4}\right)$ is related to $\mathcal{Z}\left(\kappa_{4}\right)$ by a Laplace transformation:

$$
\mathcal{Z}\left(\kappa_{4}\right)=\sum_{N_{4}} e^{-\kappa_{4} N_{4}} \mathcal{Z}\left(N_{4}\right)
$$

The phase diagram now depends on $\kappa_{0}$ and $\Delta$ and we refer to [3, 51-53] for a detailed discussion. Here we will be working in the interesting, so-called de Sitter phase where we, for a given (large) $N_{4}$ and sufficient large $t_{\text {tot }}$, observe a (Euclidean) de Sitter universe, i.e. a four-sphere where the temporal extension is proportional to $N_{4}^{1 / 4}$ while the rest of the time extension (assuming $t_{\text {tot }}$ is large enough compared to $N_{4}^{1 / 4}$ ) is a stalk of almost no spatial extension. Presumably this stalk only exists because our computer algorithm does not allow the spatial extension to shrink to zero. In figure 1 we have shown a typical situation with $t_{\text {tot }}=80, N^{(4,1)}=160000$ and we observe a bulk region (the "blob", approximately from $t=20$ to $t=60$ ) where $n_{t}$, the three-volume, i.e. the number of tetrahedra at the time-slice $t$, is large, and the rest is the stalk region where $n_{t}$ is very small. Figure 1 shows both the average over many configurations and a typical configuration which appears in the path integral. When taking the average over many configuration we align the center of mass of the blobs (see $[49,50]$ for a detailed discussion).

We can also measure the probability distribution $P^{t_{\text {tot }}}\left(n_{t}\right)$ of $n_{t}$ in the blob for a given $t$ in figure 1. It is shown in figure 2 (left figure). It is well approximated by a Gaussian distribution around the mean value $\left\langle n_{t}\right\rangle$. This is in contrast to the situation in the stalk where the probability distribution splits in three families [55], as shown on the right part of figure 2 .

Figures 1 and 2 are based on computer simulations of the type mentioned above: $N^{(4,1)}$ is kept fixed. Technically this has been done by adding a term $\varepsilon\left(N^{(4,1)}-\bar{N}^{(4,1)}\right)^{2}$ to the action, $\varepsilon$ being a suitably small parameter:

$$
S_{R} \rightarrow S_{R}+\varepsilon\left(N^{(4,1)}-\bar{N}^{(4,1)}\right)^{2} .
$$

This term ensures that $N^{(4,1)}$ is going to fluctuate not too far from $\bar{N}^{(4,1)}$. The precise value $\left\langle N^{(4,1)}\right\rangle$ depends on the choice of $\kappa_{4}$. We now want to study the transfer matrix. However, the structure of the transfer matrix is incompatible with a global constraint of this type, so we have to change the updating procedure. We have done this in two different ways. The first way is to drop the constraint term $\varepsilon\left(N^{(4,1)}-\bar{N}^{(4,1)}\right)^{2}$ and only use the discretized Einstein-Hilbert action (3.1). The way to obtain an average $\left\langle N^{(4,1)}\right\rangle$ is to fine tune $\kappa_{4}$ to $\kappa_{4}^{\text {crit }}$. The closer $\kappa_{4}$ is to the critical value the larger $\left\langle N^{(4,1)}\right\rangle$. In practice this fine tuning can 


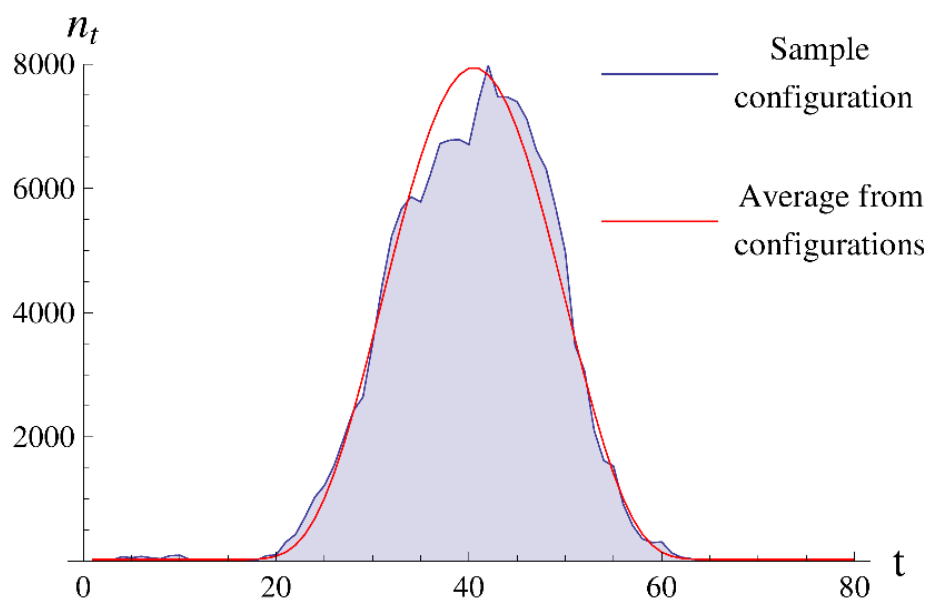

Figure 1. The distribution $n_{t}$ of the three-volume, i.e. the number of $(4,1)$-simplices, when we are in the de Sitter phase. The data is obtained for $\kappa_{0}=2.2, \Delta=0.6$. A single (typical) configuration as it appears in the path integral is shown by the blue line, and the average distribution is indicated by the red line.
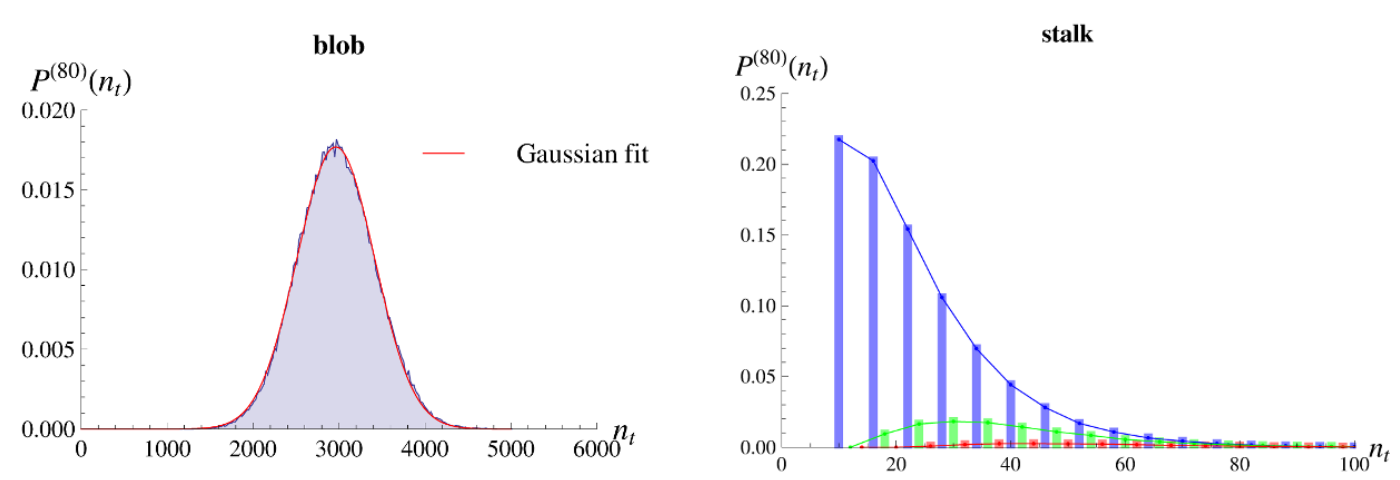

Figure 2. Left figure: The probability distribution $P^{t_{\text {tot }}}\left(n_{t}\right)$ of the volume $n_{t}$ in the blob $(t=29$ in figure 1). Right figure: The probability distribution $P^{t_{\text {tot }}}\left(n_{t}\right)$ for a typical time $t$ in the stalk (i.e. any $t$ in the range $1 \leq t \leq 17$ or $63 \leq t \leq 80$ in figure 1$)$. In the stalk the probability distribution separates into three distinct distribution corresponding to $n_{t}=(10,16,22, \ldots), n_{t}=(12,18,24, \ldots)$ and $n_{t}=(14,20,26, \ldots)$ (by construction $n_{t}$ is always even).

be difficult and the larger the system the more difficult the fine tuning. Thus we can and will only use it for small systems. For larger systems we apply a different strategy which also constrains the value of $N^{(4,1)}$, but which is compatible with the transfer matrix structure: we change the global constraint imposed on $N^{(4,1)}$ in (3.4) to a local constraint in $t$ :

$$
S_{R} \rightarrow \tilde{S}_{R}=S_{R}+\varepsilon \sum_{t=1}^{t_{\mathrm{tot}}}\left(n_{t}-n_{\mathrm{vol}}\right)^{2}
$$

Of course this constraint will drastically change the profile $\left\langle n_{t}\right\rangle$, since $n_{t}$ will now fluctuate around $n_{\mathrm{vol}}$. Thus we will have different transfer matrices $\tilde{\mathcal{M}}$ and $\tilde{M}$, and different 


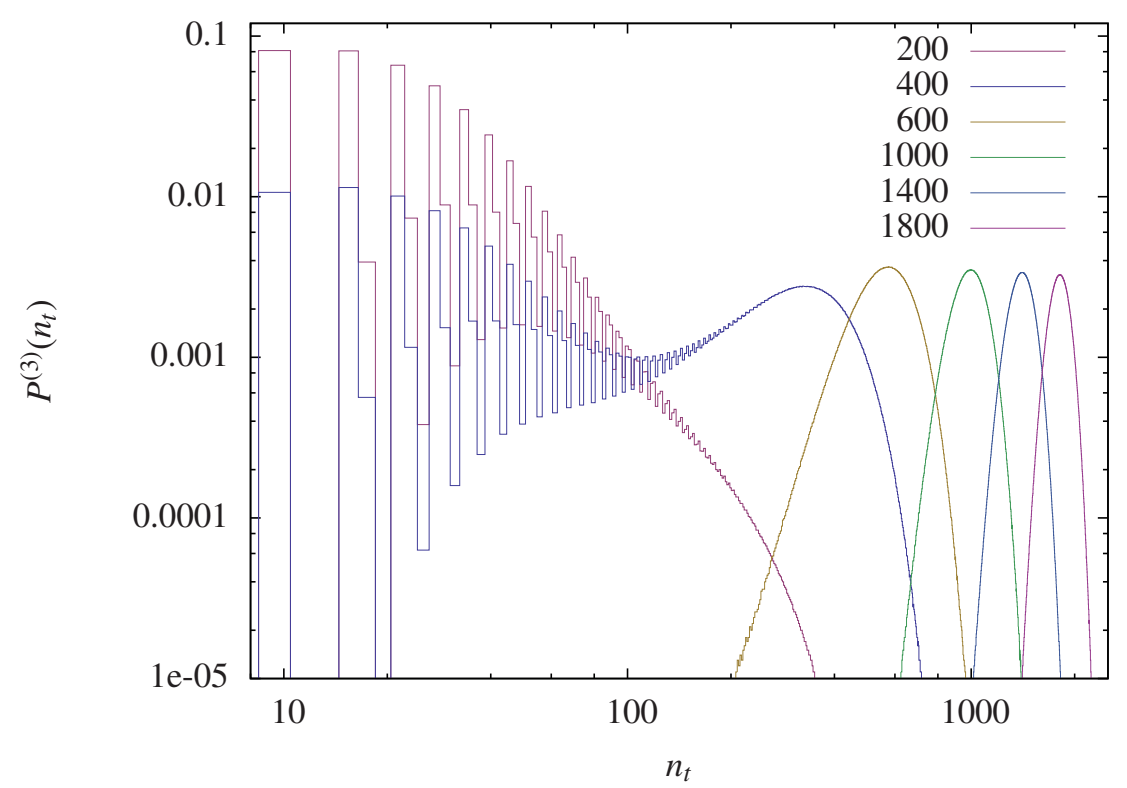

Figure 3. Probability distribution of the volume $n_{t}$, for various $n_{\mathrm{vol}}=200,400,600,1000,1400$ and 1800 (from left to right). For all ranges, the simulations were performed with $\kappa_{4}=0.322$ and $\epsilon=0.00002$.

probability distributions $\tilde{P}\left(n_{1}, n_{2}, \ldots\right)$. The new probability distribution for $n_{t}$ is shown for various $n_{\mathrm{vol}}$ in figure 3 .

However, we can reconstruct some of the probability distributions associated with the action $S_{R}$ if we know it (i.e. measure it) for the action $\tilde{S}_{R}$. The probability for measuring $\left(n_{1}, n_{2}, \ldots, n_{t_{\text {tot }}}\right)$ is given by

$$
\tilde{P}^{t_{\text {tot }}}\left(n_{1}, n_{2}, \ldots, n_{t_{\text {tot }}}\right)=\frac{\left\langle n_{1}|\tilde{M}| n_{2}\right\rangle\left\langle n_{2}|\tilde{M}| n_{3}\right\rangle \cdots\left\langle n_{t_{\text {tot }}}|\tilde{M}| n_{1}\right\rangle}{\operatorname{tr} \tilde{M}^{t_{\text {tot }}}}
$$

and is directly related to the distribution without the volume fixing term,

$$
\tilde{P}^{t_{\mathrm{tot}}}\left(n_{1}, n_{2}, \ldots, n_{t_{\mathrm{tot}}}\right) \propto P^{t_{\mathrm{tot}}}\left(n_{1}, n_{2}, \ldots, n_{t_{\mathrm{tot}}}\right) e^{-\epsilon\left(n_{1}-n_{\mathrm{vol}}\right)^{2}} \cdots e^{-\epsilon\left(n_{t_{\mathrm{tot}}}-n_{\mathrm{vol}}\right)^{2}} .
$$

We calculate the transfer matrix $\tilde{M}$ in the same way as $M$ :

$$
\langle n|\tilde{M}| m\rangle=\frac{\tilde{P}^{(3)}\left(n_{1}=n, n_{2}=m\right)}{\sqrt{\tilde{P}^{(4)}\left(n_{1}=n, n_{3}=m\right)}} .
$$

To calculate the original transfer matrix $M$ we have to cancel the volume fixing term, which is easily done: From equations (3.5), (3.6) and (3.7) we obtain

$$
\langle n|M| m\rangle=e^{\frac{1}{2} \epsilon\left(n-n_{\mathrm{vol}}\right)^{2}}\langle n|\tilde{M}| m\rangle e^{\frac{1}{2} \epsilon\left(m-n_{\mathrm{vol}}\right)^{2}} .
$$

For each choice of $n_{\mathrm{vol}}$ we observe $n_{t}$ with some approximate Gaussian distribution centered around $n_{\mathrm{vol}}$, where the width depends on our choice of $\varepsilon$, and we use the associated probabilities to construct $\langle n|M| m\rangle$, as described above. To reconstruct the matrix 

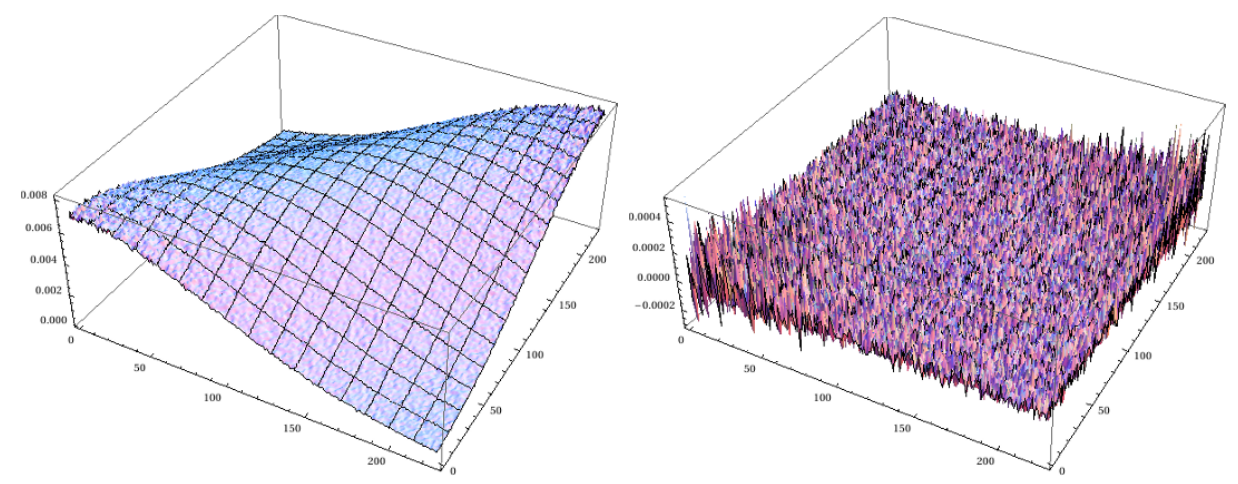

Figure 4. The left figure: The transfer matrix $M$ for range $1200<n_{t}<1600$. The matrix is optically indistinguishable from the fitted theoretical transfer matrix $M^{(t h)}$. The right figure: The difference between $M$ and $M^{(t h)}$ disappears in the numerical noise. The measurements were performed for $\kappa_{0}=2.2, \Delta=0.6, \kappa_{4}=0.9220$ and $\epsilon=0.00002$.

in a larger region of the $n$-space we have to merge data from different $n_{\text {vol }}$ regions. Since the matrix is determined only up to a normalization, the way to do this is to make sure there are regions of overlap between the $n_{t}$ distributions and in these regions choose a suitable calibration procedure such that we can merge the data. We will later describe how this is explicitly done. Needless to say the procedure we are employing here is a kind of multi-canonical Monte Carlo method (see [56] for a review).

\section{The effective action at large three-volumes}

We can measure the transfer matrix $\langle n|M| m\rangle$ for large $n, m$ as described above. As already noted it is well approximated by the matrix $M^{(t h)}$ :

$$
\left\langle n\left|M^{(t h)}\right| m\right\rangle=\mathcal{N} e^{-L_{\text {eff }}(n, m)},
$$

where the effective Lagrangian is

$$
L_{\mathrm{eff}}(n, m)=\frac{1}{\Gamma}\left[\frac{(n-m)^{2}}{n+m-2 n_{0}}+\mu\left(\frac{n+m}{2}\right)^{1 / 3}-\lambda\left(\frac{n+m}{2}\right)\right] .
$$

We now ask how well? We make a best fit of the parameters $\Gamma, \mu, \lambda$ and $\mathcal{N}$ (fixing $n_{0}=0$ ). The measured $M, M^{(t h)}$ from (4.1) as well as their difference, are shown in figure 4 for $n_{\mathrm{vol}}=1400$ and for the $n_{t}$ range $1200<n_{t}<1600$.

The values of parameters $\Gamma, \mu$ and $\lambda$ for different values of $n_{\mathrm{vol}}$, obtained from the best fits of the matrix $M^{(t h)}$ (4.1) to the measured matrix $M$ are presented in table 1. Again $n_{0}$ is chosen to be zero.

\subsection{The kinetic term}

To get a better estimation of the parameters associated with the effective action (4.2), we first try to fit only to the parameters of the kinetic term which is by far the dominating 


\begin{tabular}{|c|c|c|c|c|}
\hline$n_{\text {vol }}$ & $n_{t}$ range & $\Gamma$ & $\mu$ & $\lambda$ \\
\hline 600 & $400-820$ & $25.71 \pm 0.01$ & $18 \pm 1$ & $0.05 \pm 0.01$ \\
\hline 1000 & $780-1220$ & $26.00 \pm 0.01$ & $17 \pm 1$ & $0.05 \pm 0.01$ \\
\hline 1400 & $1180-1630$ & $26.10 \pm 0.01$ & $13 \pm 1$ & $0.04 \pm 0.01$ \\
\hline 1800 & $1580-2040$ & $26.08 \pm 0.01$ & $26 \pm 1$ & $0.07 \pm 0.01$ \\
\hline 2200 & $1980-2440$ & $26.05 \pm 0.01$ & $19 \pm 2$ & $0.05 \pm 0.01$ \\
\hline
\end{tabular}

Table 1. The values of $\Gamma, \mu$ and $\lambda$ for different $n_{\mathrm{vol}}$, obtained from best fits of $M^{(t h)}$ to the measured $M$.

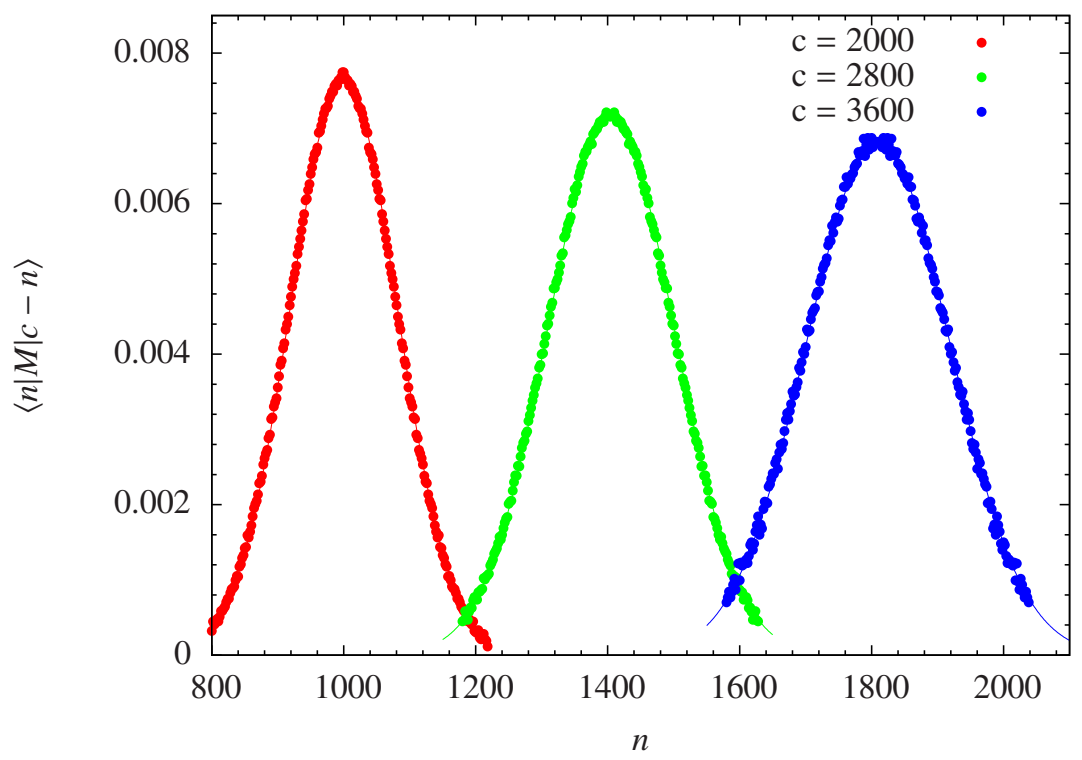

Figure 5. $\langle n|M| c-n\rangle$ plotted as a function of $n$ for $c=2000,2800$ and 3600 (dots). Gaussian fits are drawn with a line.

term from a numerical point of view. We do that by keeping the sum of the entries, i.e. $n+m$, fixed such that the potential term is not changing. In this way we can try to determine $\Gamma$ and even $n_{0}$ which we had put to zero in the fits mentioned above in order not to have too many fit-parameters. The matrix elements for constant $n+m=c$ show the expected Gaussian dependence on $n$ (see figure 5):

$$
\langle n|M| m\rangle=\langle n|M| c-n\rangle=\mathcal{N}(c) \exp \left[-\frac{(2 n-c)^{2}}{\Gamma \cdot\left(c-2 n_{0}\right)}\right]
$$

where the terms in the effective action which only depend on $c$ are included in the normalization.

We expect the denominator of the kinetic term $k(c)$ to behave like $k(n+m)=\Gamma$. $\left(n+m-2 n_{0}\right)$. As shown on figure 6 this is indeed true and the parameter $\Gamma$ is common 


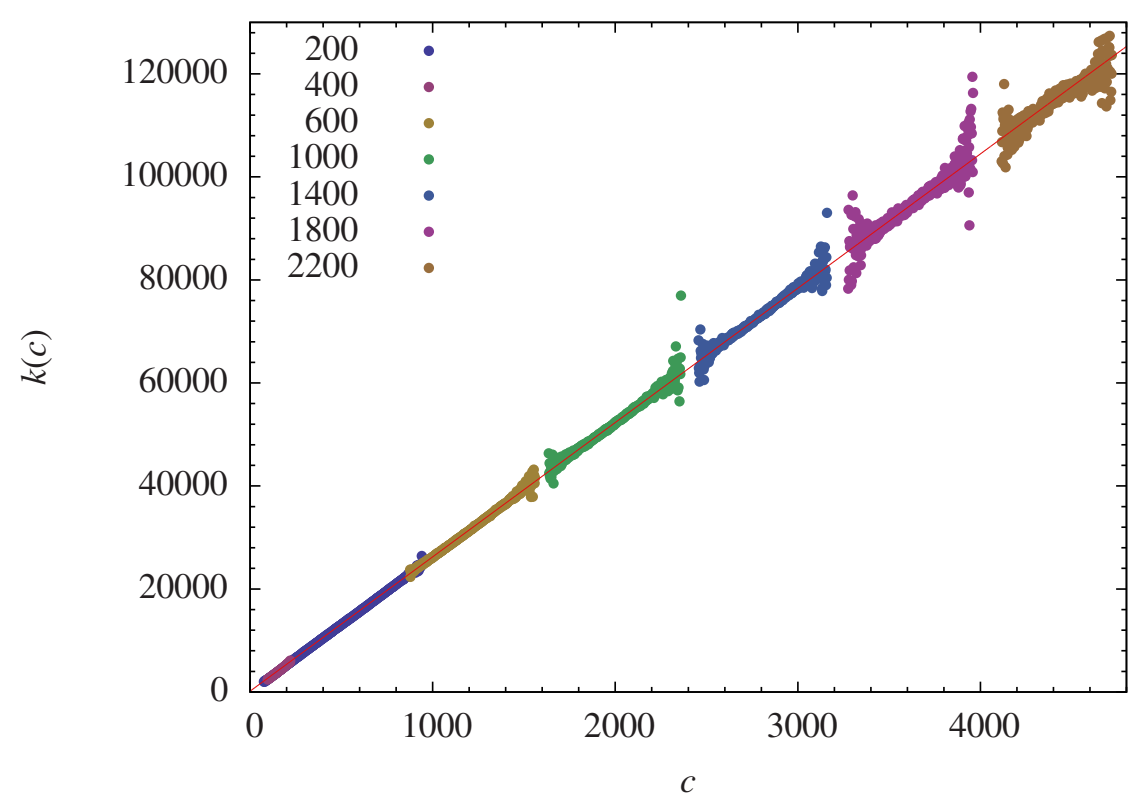

Figure 6. The coefficient $k(c)$ in the kinetic term as a function of $c=n+m$ (different colors denote different ranges), and a linear fit $k(n+m)=\Gamma \cdot\left(n+m-2 n_{0}\right)$ (red line).

for all ranges. Figure 6 presents measured coefficients $k(c)$ for various $c$ 's and ranges of $n_{t}$ denoted by distinct colors together with a linear fit. The best linear fit gives $\Gamma=26.07$ and $n_{0}=-3$, which is consistent with results obtained for separate ranges of $n_{t}$.

\subsection{The potential term}

The potential part of the effective Lagrangian may be extracted from the diagonal elements of the transfer matrix

$$
L_{\mathrm{eff}}(n, n)=-\log \langle n|M| n\rangle+c\left(n_{\mathrm{vol}}\right)=\frac{1}{\Gamma}\left(\mu n^{1 / 3}-\lambda n\right) .
$$

However, because of different normalizations of the transfer matrices for different ranges (hence the dependence of the constant $c\left(n_{\mathrm{vol}}\right)$ on $\left.n_{\mathrm{vol}}\right)$, the fit of $L_{\mathrm{eff}}(n, n)$ to the transfer matrix data cannot be performed in a straightforward way. The transfer matrices have first to be merged properly via a scaling procedure, i.e. by adjusting the $c$ constant in (4.4). This is done in the following way. For example, for $n_{\mathrm{vol}}=1400$ the range of spatial volumes for which we measured the transfer matrix is $n_{t}=1180 \ldots 1630$, while for $n_{\mathrm{vol}}=1800$ the range is $n_{t}=1580 \ldots 2040$. Thus, there is a non-vanishing intersection $n_{t}=1580 \ldots 1630$ for which elements of both matrices were measured. We scale the second matrix, so that the mean value of the diagonal elements on the intersecting region is equal for both matrices. After applying this procedure for successive ranges, we finally get scaled transfer matrices which can be merged. The result of such merging is shown on figure 7 , which shows the diagonal elements of the scaled transfer matrices together with a fit of form (4.4), where we took $\Gamma=26.1$. 


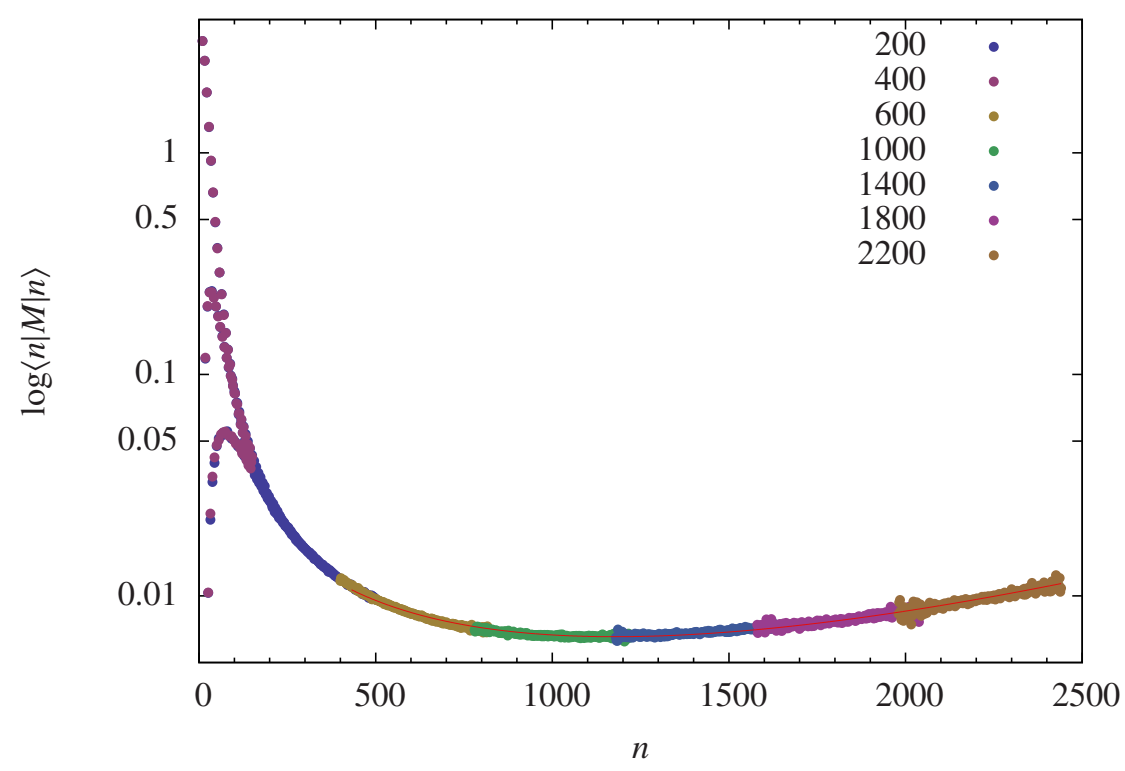

Figure 7. $\log \langle n|M| n\rangle$ of the scaled transfer matrix (dots, different colors denote different ranges) compared with the fit of the potential term $-L_{\text {eff }}$ (red line, which stops at $n=400$ ).

\begin{tabular}{|c|c|c|c|c|}
\hline Method & $\Gamma$ & $n_{0}$ & $\mu$ & $\lambda$ \\
\hline Cross-diagonals & $26.07 \pm 0.02$ & $-3 \pm 1$ & - & - \\
\hline Diagonal & $(26.07)$ & - & $16.5 \pm 0.2$ & $0.049 \pm 0.001$ \\
\hline Full fit & $26.17 \pm 0.01$ & $7 \pm 1$ & $15.0 \pm 0.1$ & $0.046 \pm 0.001$ \\
\hline Previous method* $^{*}$ & $23 \pm 1$ & - & $13.9 \pm 0.7$ & $0.027 \pm 0.003$ \\
\hline
\end{tabular}

Table 2. The values of $\Gamma, n_{0}, \mu$ and $\lambda$ fitted in different ways. ${ }^{*}$ We also present the parameters of the effective action extracted from the covariance matrix of volume fluctuations in our earlier work [55].

\subsection{A global effective action fit}

Summing up, the effective action determined via the transfer matrices is strikingly well described by eq. (4.2). As in the last subsection we can merge the scaled matrices for all the choices of $n_{\mathrm{vol}}$ (see figure 8), and fit expression (4.2) to the aggregated data. The best fit gives $\Gamma=26.17, n_{0}=7, \mu=15.0$ and $\lambda=0.046$. We summarize the results of fitting the parameters of $L_{\text {eff }}$ in different ways in table 2. As long as we are concentrating on the large (bulk) values of $n_{t}$ the various data clearly do not allow us to improve the expression (4.2).

For comparison we also present the parameters of the effective action measured indirectly from the covariance matrix of volume fluctuations. This method is based on the analysis of the effective propagator around the semi-classical solution [55]. The parameters of the action from the previous method agree quite well with those measured directly from the transfer matrix. The small difference may result from slightly different parametrization of the potential term in the effective action (see footnote 3 ). 


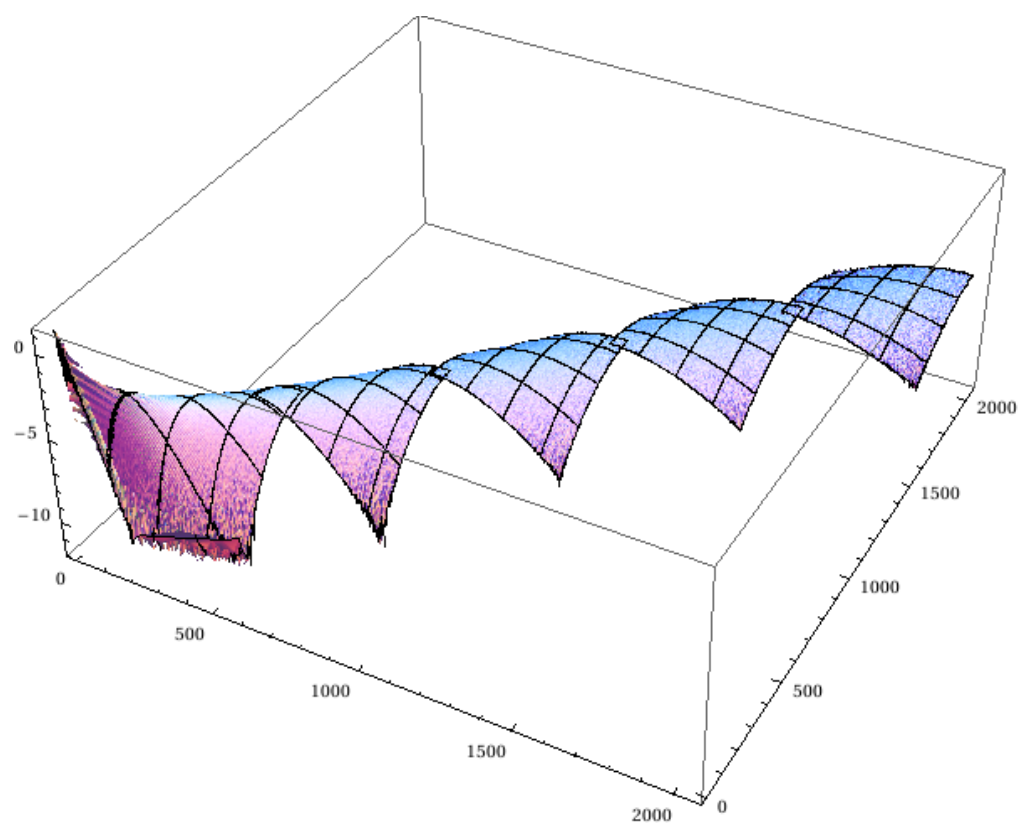

Figure 8. Logarithm of merged and scaled transfer matrices measured for different $n_{\mathrm{vol}}$.

\begin{tabular}{|c|c|c|}
\hline$i$ & $\lambda_{i}$ & $\lambda_{i}^{(t h)}$ \\
\hline 1 & $1.168 \times 10^{0}$ & $1.168 \times 10^{0}$ \\
\hline 2 & $3.824 \times 10^{-1}$ & $3.828 \times 10^{-1}$ \\
\hline 3 & $7.061 \times 10^{-2}$ & $7.074 \times 10^{-2}$ \\
\hline 4 & $8.543 \times 10^{-3}$ & $8.640 \times 10^{-3}$ \\
\hline 5 & $-1.516 \times 10^{-3}$ & $7.857 \times 10^{-4}$ \\
\hline 6 & $1.507 \times 10^{-3}$ & $5.715 \times 10^{-5}$ \\
\hline
\end{tabular}

Table 3. The first 6 eigenvalues of the measured transfer matrix $\langle n|M| m\rangle$ calculated for $n_{\mathrm{vol}}=1400$ and the similar eigenvalues for the fitted transfer matrix $\left\langle n\left|M^{(t h)}\right| m\right\rangle$.

\subsection{Miscellaneous}

The spectral decomposition of matrices presents us with an interesting way to compare the measured transfer matrix $\langle n|M| m\rangle$ with the "theoretical" matrix $\left\langle n\left|M^{(t h)}\right| m\right\rangle$ given by eq. (4.1) with parameters obtained from a best fit, as described above (in this case the fit from the $n_{\mathrm{vol}}=1400$ data). We obtain very good agreement between eigenvalues and eigenvectors for the two matrices. The first 6 eigenvalues are listed in table 3 and the first 4 eigenvectors are shown in figure 9 .

As a final check of the consistency of the effective transfer matrix with data we measure the probability distribution of a spatial slice volume $\tilde{P}^{\left(t_{\text {tot }}\right)}(n)$ in terms of the transfer 

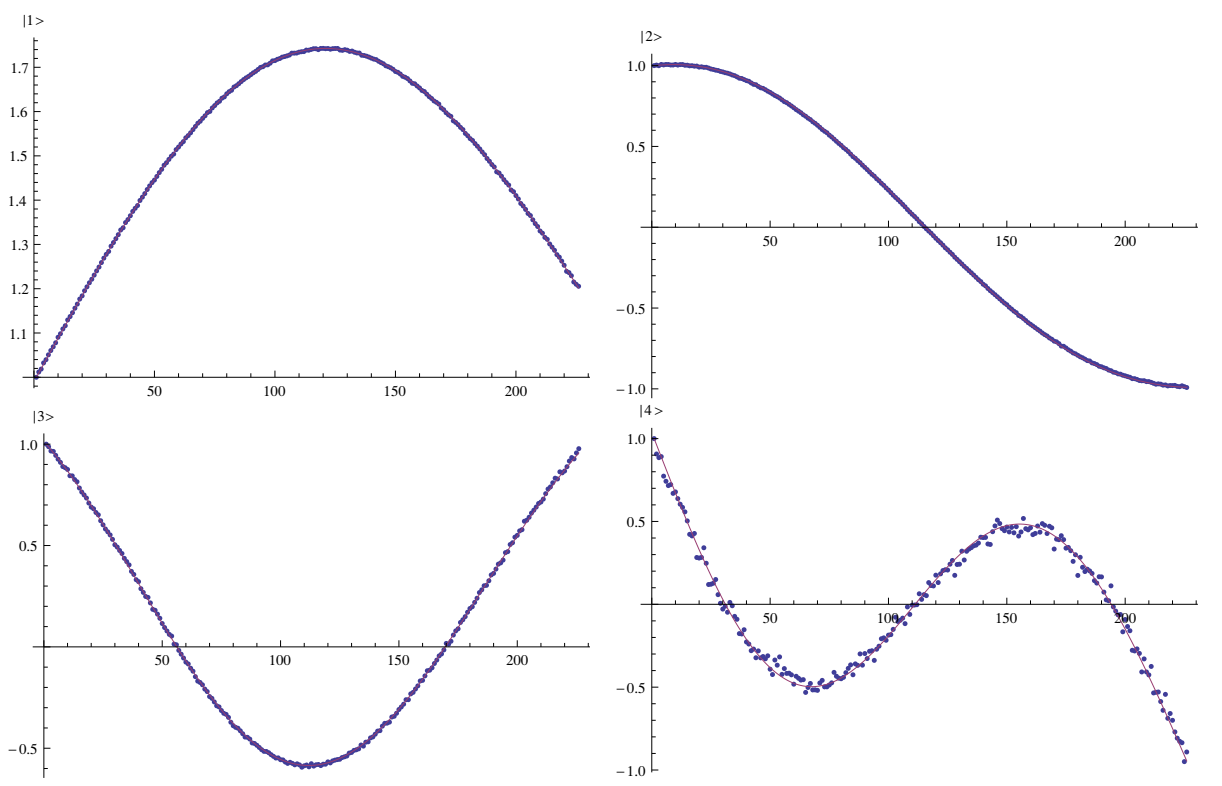

Figure 9. The first 4 eigenvectors of the measured transfer matrix $\langle n|M| m\rangle$ (calculated for $n_{\mathrm{vol}}=1400($ dots $\left.)\right)$, and the first 4 eigenvectors of the corresponding theoretical transfer matrix $\left\langle n\left|M^{(t h)}\right| m\right\rangle$ (lines).
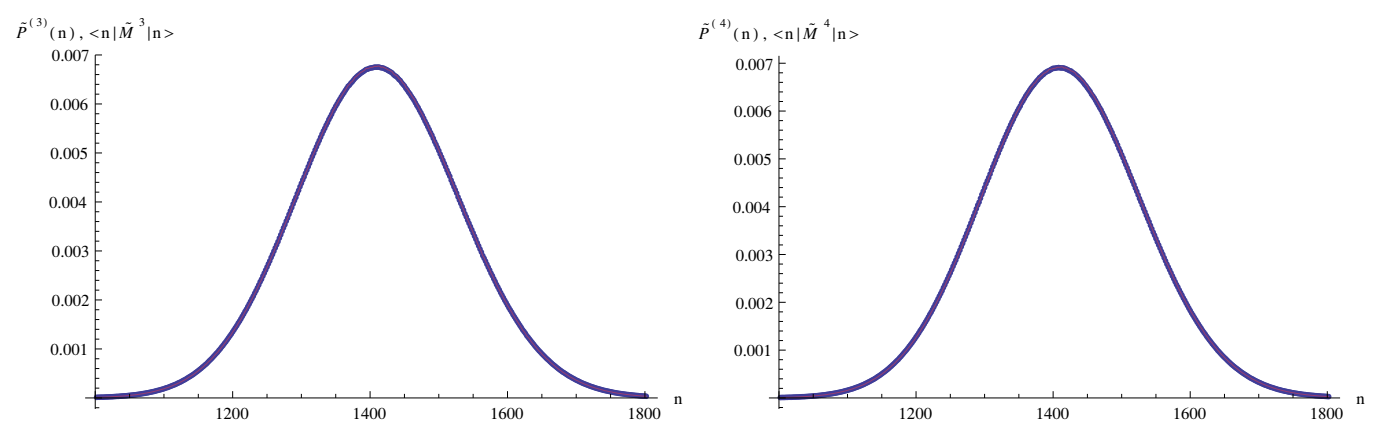

Figure 10. The probability distribution $\tilde{P}^{t_{\text {tot }}}(n)$ (dots) and $\frac{\left\langle n\left|\tilde{M}^{t_{\text {tot }}}\right| n\right\rangle}{\operatorname{tr} \tilde{M}^{t_{\text {tot }}}}$ (line) for $t_{\text {tot }}=3$ (left) and $t_{\text {tot }}=4$ (right).

matrix $\tilde{M}$ given by

$$
\tilde{P}^{t_{\text {tot }}}(n)=\frac{\left\langle n\left|\tilde{M}^{t_{\text {tot }}}\right| n\right\rangle}{\operatorname{tr} \tilde{M}^{t_{\text {tot }}}}
$$

We use $\tilde{M}$ instead $M$, because the power $M^{t_{\text {tot }}}$ involves summation over all possible volumes $n$ which are not accessible until we suppress them with the term $e^{-\epsilon\left(n-n_{\mathrm{vol}}\right)^{2}}($ which is present in $\tilde{M})$. We compare the theoretical expectation $(4.5)$ with the measured $\tilde{P}^{t_{\text {tot }}}(n)$ for $t_{\text {tot }}=3$ and $t_{\text {tot }}=4$. The comparison is shown on figure 10 and the error is of order $0.02 \%$. 


\section{The transfer matrix for small three-volumes}

We have seen that the effective transfer matrix is very well described by the simplest effective Lagrangian (4.2). However, it is natural to expect that the corresponding effective action is only a first approximation. Appealing to isotropy and homogeneity one would expect that a potential $R^{k}(t)$ term, where $R(t)$ refers to the scalar curvature of the threedimensional space at time $t$, translates into a term $\left(\frac{n_{t}+n_{t+1}}{2}\right)^{1-2 k / 3}$. The leading term $R(t)$ is already part of the effective action where it appears as the term $\left(\frac{n_{t}+n_{t+1}}{2}\right)^{1 / 3}$. For the data coming from large $n_{t}$ we have seen that the difference between our measured $M$ and $M^{(t h)}$ coming from the effective action with only the $\left(\frac{n_{t}+n_{t+1}}{2}\right)^{1 / 3}$ term is already at the noise level. From these large $n_{t}$ data we have no chance with the present statistics to study higher $k$ corrections. Thus we now turn to the small $n_{t}$ region. The difficulty of analyzing the small $n_{t}$ region is that we might encounter discretization effects as is apparent from figure 2. The study of this region started in [55], but the present approach offers the great advantage that we can perform high statistics study of small systems, while in the earlier studies the interesting region was a small part of a larger system and thus the statistics becomes less good.

We want to measure the effective transfer matrix by measuring $P^{t_{\text {tot }}}\left(n_{i}, n_{j}\right)$ for $t_{\text {tot }}=$ 3,4 as we have already done, but now for small $n_{t}$, i.e. for much smaller systems. We also want to check as well as possible that the concept of an "effective" transfer matrix actually works. While it seemed to work well for large $n_{t}$, it is not obvious that this will remain true for small $n_{t}$ : physics might be different and discretization effects might also spoil such a picture. Our systems will be so small that we do not have to introduce the auxiliary transfer matrix $\tilde{M}$ and the volume fixing parameter $n_{\mathrm{vol}}$. Thus we only use the Regge Einstein-Hilbert action $S_{R}$, eq. (3.1), to generate the probability distributions $P^{(3)}\left(n_{1}, n_{2}\right)$ and $P^{(4)}\left(n_{1}, n_{3}\right)$ and construct $\langle n|M| m\rangle$ from eq. (2.18).

\subsection{Eigenvectors analysis}

Given the transfer matrix $M$ we can perform a spectral decomposition in terms of eigenvalues $\lambda_{i}$ and (orthonormal) eigenvectors $\left|\alpha_{i}\right\rangle$ :

$$
M=\sum_{i} \lambda_{i}\left|\alpha_{i}\right\rangle\left\langle\alpha_{i}\right|
$$

Since the measured $M$ is only determined up to a normalization, we will assume $\lambda_{1}=1$ and $\left|\lambda_{1}\right| \geq\left|\lambda_{2}\right| \geq \ldots$

If the gaps are significant between the first, second and third eigenvalues, it is clear that the large $t_{\text {tot }}$ limit of $\mathcal{Z}\left(t_{\text {tot }}\right)$ and the large $\Delta t$ limit of the simplest correlation functions $P^{t_{\text {tot }}}\left(n_{t}, n_{t+\Delta t}\right)$ will be completely dominated by the first two eigenstates: Thus, in the limit where $t_{\text {tot }} \gg \Delta t \gg 1$ we have (recalling that $\lambda_{1}$ is normalized to 1 )

$$
\mathcal{Z}\left(t_{\mathrm{tot}}\right)=\sum_{i}\left(\lambda_{i}\right)^{t_{\mathrm{tot}}} \approx 1
$$




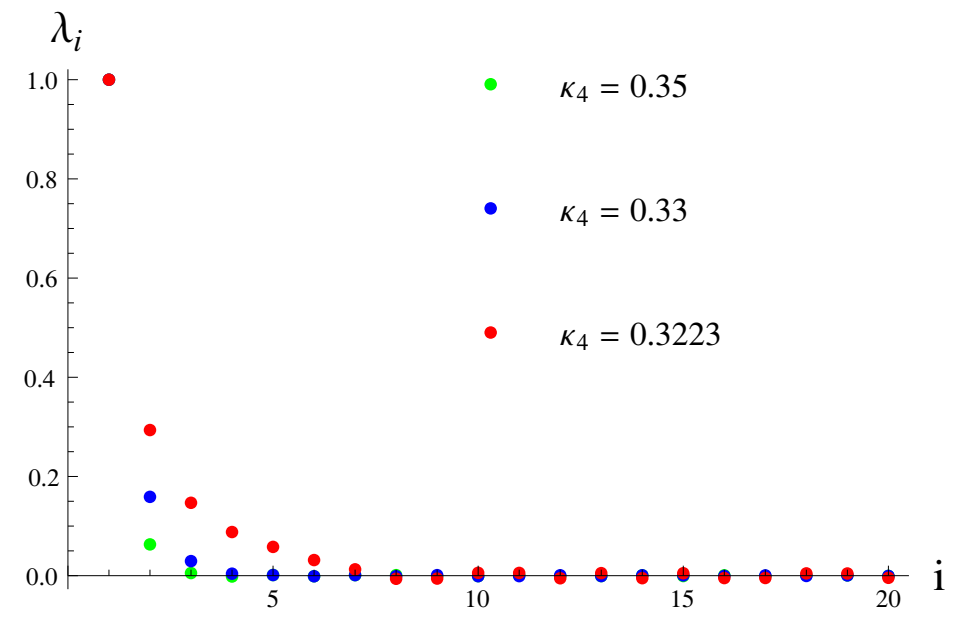

Figure 11. Eigenvalues of the transfer matrix $M$ for different values of $\kappa_{4}$ approaching $\kappa_{4}^{\text {crit }} \approx$ 0.3222. Note that according to our normalization the biggest eigenvalue is set to one.

$$
\begin{aligned}
P^{t_{\mathrm{tot}}}(n) & =\frac{1}{Z} \sum_{i} \lambda_{i}^{t_{\mathrm{tot}}}\left\langle n \mid \alpha_{i}\right\rangle^{2} \approx\left\langle n \mid \alpha_{1}\right\rangle^{2} \\
P^{t_{\mathrm{tot}}}\left(n_{t}, m_{t+\Delta t}\right) & =\frac{1}{Z}\left(\sum_{i}\left(\lambda_{i}\right)^{\Delta t}\left\langle n \mid \alpha_{i}\right\rangle\left\langle\alpha_{i} \mid m\right\rangle\right)\left(\sum_{i}\left(\lambda_{i}\right)^{t_{\mathrm{tot}}-\Delta t}\left\langle n \mid \alpha_{i}\right\rangle\left\langle\alpha_{i} \mid m\right\rangle\right) \\
& \approx\left\langle n \mid \alpha_{1}\right\rangle^{2}\left\langle\alpha_{1} \mid m\right\rangle^{2}+\lambda_{2}^{\Delta t}\left\langle n \mid \alpha_{2}\right\rangle\left\langle\alpha_{2} \mid m\right\rangle\left\langle n \mid \alpha_{1}\right\rangle\left\langle\alpha_{1} \mid m\right\rangle
\end{aligned}
$$

The average can be written as:

$$
\langle n\rangle_{t_{\mathrm{tot}}} \approx \sum_{n} n\left\langle n \mid \alpha_{1}\right\rangle^{2}
$$

For the correlator we have:

$$
\left\langle n_{t} m_{t+\Delta t}\right\rangle-\left\langle n_{t}\right\rangle\left\langle m_{t+\Delta t}\right\rangle \approx \lambda_{2}^{\Delta t} \sum_{n, m} n m\left\langle n \mid \alpha_{2}\right\rangle\left\langle\alpha_{2} \mid m\right\rangle\left\langle n \mid \alpha_{1}\right\rangle\left\langle\alpha_{1} \mid m\right\rangle .
$$

and the long distance behavior is an exponential fall off $e^{-\mu \Delta t}, \mu=-\log \lambda_{2} / \lambda_{1}$ (where we have reintroduced $\lambda_{1}$ for clarity).

How well are these approximate relations satisfied? First we observe that there is indeed a clear gap between the first eigenvalues, as illustrated in figure 11. The closer $\kappa_{4}$ is fine-tuned to $\kappa_{4}^{\text {crit }}$ the smaller the gap, but even very close to the critical value we observe a clear gap. That we have gaps even at $\kappa_{4}^{\text {crit }}$ just illustrates that we indeed consider small systems.

Next we ask how large $t_{\text {tot }}$ has to be in order that the approximations made in eq. (5.3)(5.5) are valid. That can of course be read off from the eigenvalues and already for $t_{\text {tot }}=4$ the approximation is very good for $\langle n\rangle_{t_{\text {tot }}}$. For the correlator one has of course to consider larger $t_{\text {tot }}$. In figure 12 we have shown for $t_{\text {tot }}=12$ the expected exponential decay with exponent $\log \left(\lambda_{2} / \lambda_{1}\right)$ compared to the actually measured correlator. The agreement is very good even for small $\Delta t$ where it is not obvious that ignoring the eigenvectors with eigenvalues smaller than $\lambda_{2}$ is a valid approximation. 


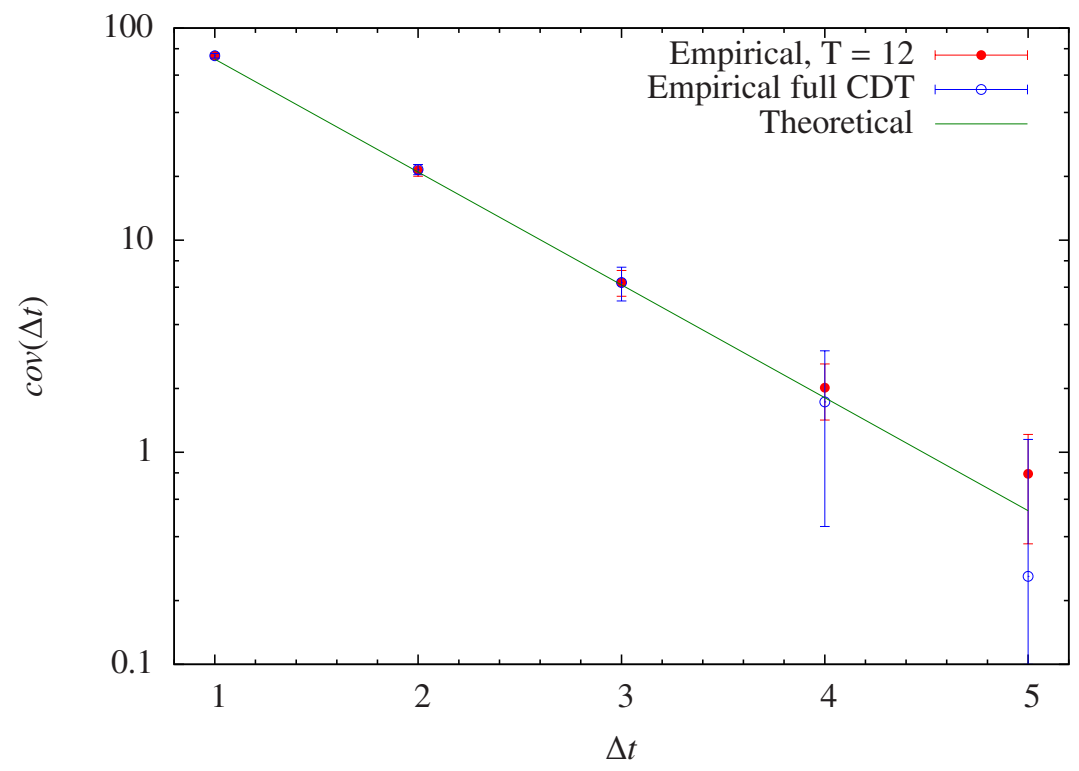

Figure 12. The "theoretical" correlator (5.5) of spatial volumes between time-slices separated by $\Delta t$ and calculated including the first two eigenvectors of the transfer matrix $M$ calculated for $\kappa_{4}=0.3223$ (green line). The correlator decays exponentially with $\Delta t$ (log scale). There is a very good agreement with the correlator measured directly in simulations for $\mathrm{T}=12$ (red) and in "full CDT" stalk range (blue). The bars indicate measurement errors.

\subsection{The "full-CDT" approximation}

We have also compared our approximate large $t_{\text {tot }}$ probability distributions $P(n)$ (eq. (5.3)) with the data taken from the stalk range of full CDT simulations (including the blob and the stalk range). The distributions approach very well "full CDT" measurements as $\kappa_{4}$ tends to critical value (figure 13). The validity of the transfer matrix model is further confirmed by the behaviour of the correlator $\left\langle n_{t} m_{t+\Delta t}\right\rangle-\left\langle n_{t}\right\rangle\left\langle m_{t+\Delta t}\right\rangle$ measured directly in the stalk range of "full CDT". As illustrated in figure 12 the measured correlator falls off as $e^{-\mu \Delta t}$. The parameter $\mu$ is well explained by the ratio of the first two eigenvalues of the transfer matrix $M$ calculated for $\kappa_{4}$ closest to the critical value.

\section{The effective action for small three-volumes}

In principle the matrix $M$ presented above would allow us to determine an effective Lagrangian for small $n_{t}$, i.e. even in the stalk range of the CDT configurations, precisely as we did for large $n_{t}$ :

$$
S_{\mathrm{eff}}=\sum_{t} L_{\mathrm{eff}}\left(n_{t}, n_{t+1}\right), \quad\langle n|M| m\rangle=\mathcal{N} e^{-L_{\mathrm{eff}}(n, m)} .
$$

However, we are confronted with the existence of three families of states, as is apparent in figure 2. We can however define a reduced matrix $\hat{M}$ performing a summation over the three families, i.e.

$$
\hat{M}=U M U^{T}
$$




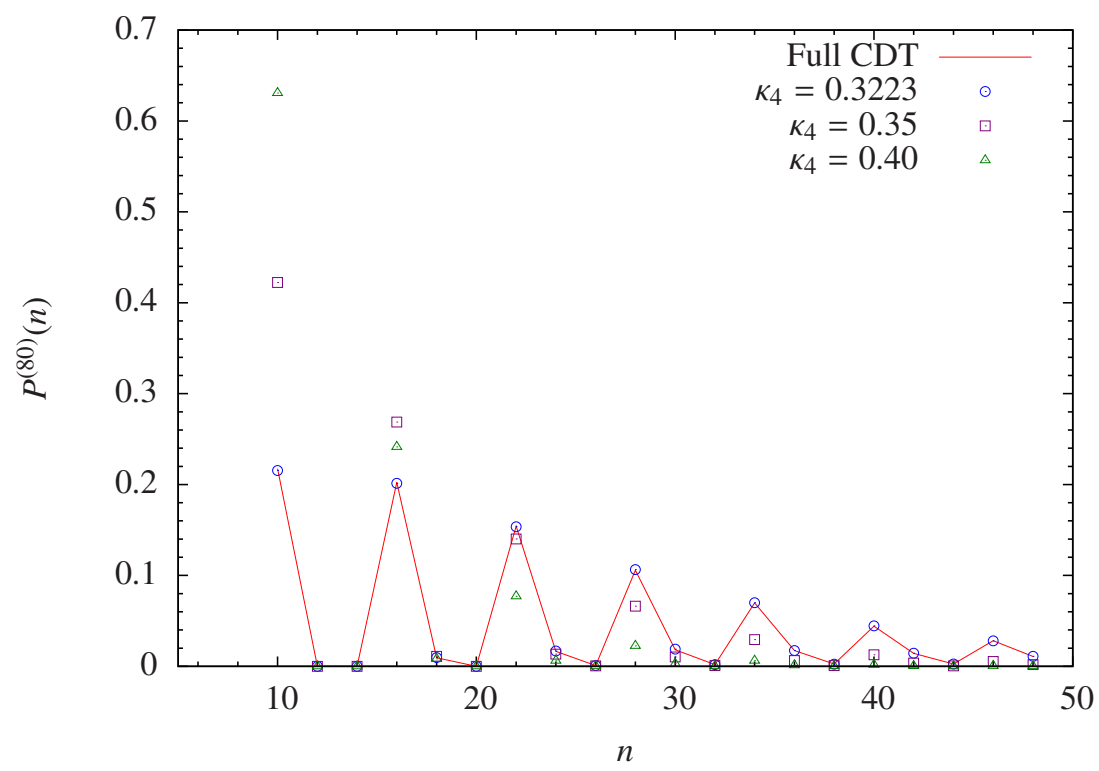

Figure 13. Theoretical 1-point probability distributions calculated with the 'largest' eigenvector of the transfer matrix $M$ approach empirical probability measured in "full CDT" (red line) as $\kappa_{4}$ tends to critical value. Theoretical distributions were computed for $\kappa_{4}=0.40,0.35$ and 0.3223 . For $\kappa_{4}=0.3223$ (blue circles) the agreement is very good.

where the rectangular matrix $U$ has a form:

$$
U=\left\{\begin{array}{cccccccccc}
1 & 1 & 1 & 0 & 0 & 0 & 0 & 0 & 0 & \cdots \\
0 & 0 & 0 & 1 & 1 & 1 & 0 & 0 & 0 & \cdots \\
0 & 0 & 0 & 0 & 0 & 0 & 1 & 1 & 1 & \cdots \\
\cdots & & & & & &
\end{array}\right\}
$$

The elements of the matrix $\hat{M}$ behave much more smoothly and can be analyzed, using the effective action idea. We normalize $\hat{M}$ by choosing its largest eigenvalue to be one.

Let us assume that $L_{\mathrm{eff}}$ has the form:

$$
L_{\mathrm{eff}}(n, m)=\frac{(n-m)^{2}}{k(n+m)}+v(n+m)
$$

where the functions $k(\cdot)$ and $v(\cdot)$ are to be determined.

We follow the same strategy as when $n_{t}$ was large: first we analyze matrix elements for constant $n+m=c$ in order to keep the potential term $v(n+m)$ constant. One observes a Gaussian dependence on $n$ (figure 14):

$$
\langle n|\hat{M}| m\rangle=\langle n|\hat{M}| c-n\rangle=\mathcal{N} \exp \left[-\frac{(2 n-c)^{2}}{k(c)}-v(c)\right]
$$




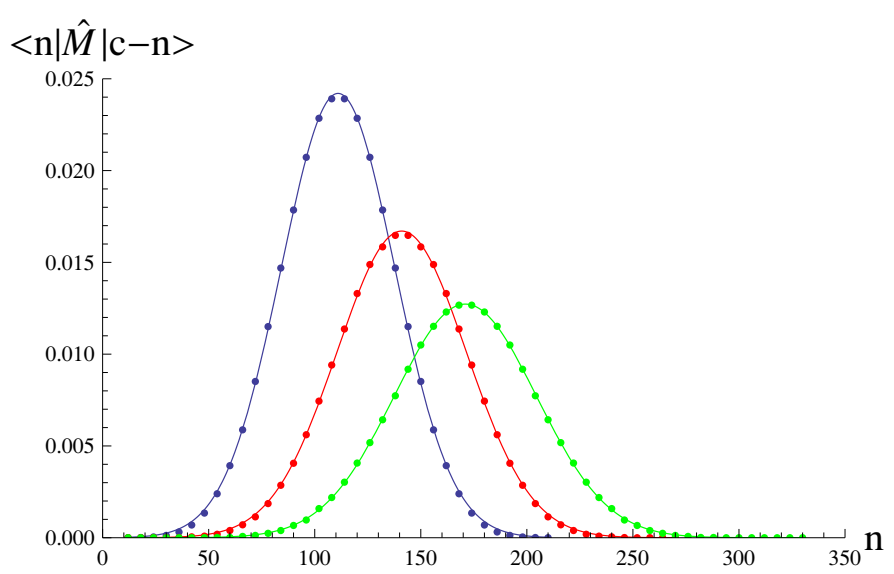

Figure 14. $\langle n|\hat{M}| c-n\rangle$ plotted as a function of $n$ for $c=222$ (blue), $c=282$ (red), $c=342$ (green). Gaussian fits (6.4) are presented as solid lines.

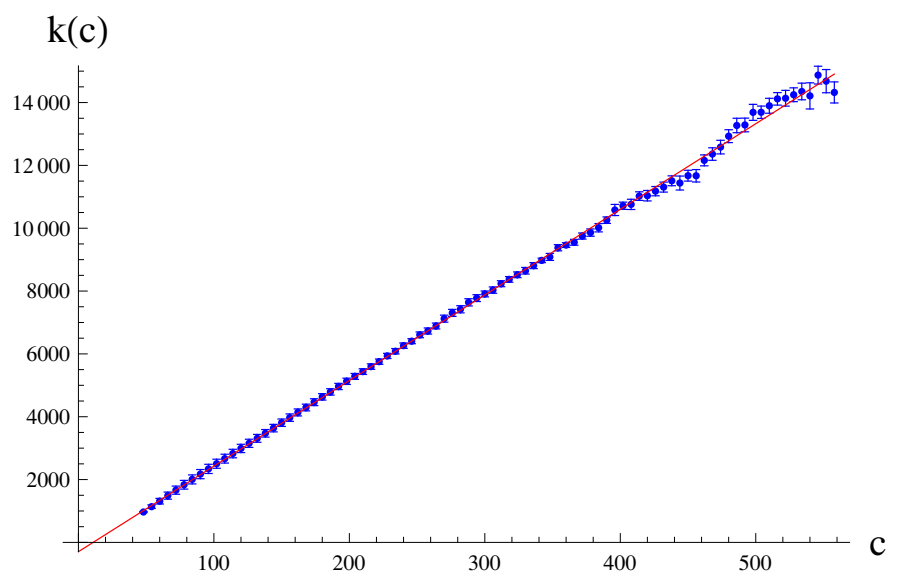

Figure 15. Kinetic coefficients $k(c)$ (blue) and a linear fit (red).

Fitting (6.4) for different $c$ 's one can easily check that the kinetic coefficient $k(c)$ is linear (figure 15). Therefore one can write:

$$
L_{\mathrm{eff}}(n, m)=\frac{1}{\Gamma}\left[\frac{(n-m)^{2}}{n+m-2 n_{0}}+\widetilde{v}(n+m)\right]
$$

where: $\widetilde{v}()=\Gamma v()$. The best fit of $\Gamma$ and $n_{0}$ is presented in table 4 .

In the above we recognize the familiar kinetic term present in the effective action for the blob range. Let us also test the assumption that the potential part is similar by analyzing the diagonal elements of $\hat{M}$ where the kinetic term is zero. From (6.1) and (6.5) one obtains

$$
\log \langle n|\hat{M}| n\rangle=-\frac{1}{\Gamma} \widetilde{v}(2 n)+\log \mathcal{N}
$$

Let us assume

$$
\widetilde{v}(2 n)=-\lambda n+\mu n^{1 / 3}+\delta n^{-\rho},
$$




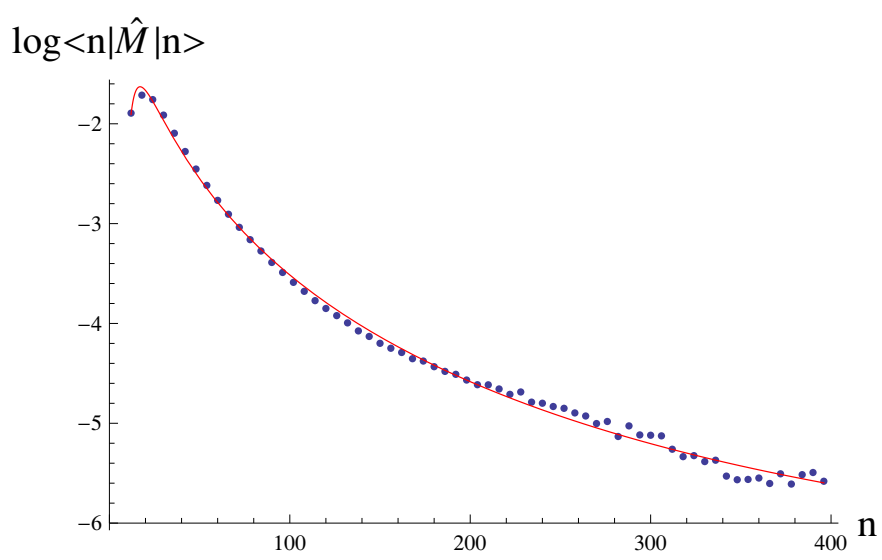

Figure 16. Logarithm of the diagonal $\langle n|\hat{M}| n\rangle$ (blue dots) compared with the fit of the potential term (6.7) (red line).

\begin{tabular}{|c|c|c|}
\hline Parameter & Stalk & Blob \\
\hline$\Gamma$ & $27.2 \pm 0.1$ & $25.7-26.2$ \\
\hline$n_{0}$ & $5 \pm 1$ & $-3-+7$ \\
\hline$\mu$ & $34 \pm 2$ & $13-30$ \\
\hline$\lambda$ & $0.12 \pm 0.02$ & $0.04-0.07$ \\
\hline$\delta$ & $(4 \pm 7) \times 10^{4}$ & - \\
\hline$\rho$ & $3 \pm 1$ & - \\
\hline
\end{tabular}

Table 4. Fitted parameters of the effective action for the stalk (6.8). For comparison we also present estimates of parameters of the effective action for the blob calculated from the large $n_{t}$ simulations (see table 1 and 2).

inspired by the bulk expressions already derived and where we have included a new term $\delta n^{-\rho}$, inspired by earlier remarks about effective powers of the three-dimensional curvature $R(t)$. We present the fit of the logarithm of the diagonal elements $\langle n|\hat{M}| n\rangle$ in figure 16 and the parameters of the fit in table 4.

It is surprising that the same effective action is still present in the stalk, even though the volume behavior seems at the first sight quite different from that in the blob range. It is even more surprising that the parameters of the fit agree quite well with the effective Newton constant $\Gamma$ measured in the blob range. The parameter $\mu$ is slightly bigger but of the same order of magnitude as the potential coefficient from the blob range. Only $\lambda$ which is related to the size of the dynamically created universe is quite different, but that should be no surprise since $\lambda$ semiclassically is related to the size of the universe. Finally, since not even the sign of $\delta$ is well determined, one can not attach too much meaning the value $\rho \approx 3$ determined by a best fit. There seems to be too many parameters for a reliable fit to the data. From the corrections coming from higher powers of $R(t)$ one expects $\rho=2 k / 3-1$, $k=2, \ldots$. We have tried to fix $\rho=1 / 3$, the first expected correction, in which case $\delta$ comes out positive, but still with large error-bars. 


\begin{tabular}{|c|c|c|}
\hline$i$ & $\lambda_{i}$ & $\lambda_{i}^{(t h)}$ \\
\hline 1 & $1.000 \times 10^{0}$ & $0.973 \times 10^{0}$ \\
\hline 2 & $3.922 \times 10^{-1}$ & $4.074 \times 10^{-1}$ \\
\hline 3 & $2.007 \times 10^{-1}$ & $2.054 \times 10^{-1}$ \\
\hline 4 & $9.308 \times 10^{-2}$ & $9.658 \times 10^{-2}$ \\
\hline 5 & $3.483 \times 10^{-2}$ & $3.745 \times 10^{-2}$ \\
\hline 6 & $1.085 \times 10^{-2}$ & $1.199 \times 10^{-2}$ \\
\hline
\end{tabular}

Table 5. The first 6 eigenvalues of the measured 'reduced' transfer matrix $\langle n|\hat{M}| m\rangle$ and the corresponding eigenvalues for the fitted transfer matrix $\left\langle n\left|\hat{M}^{(t h)}\right| m\right\rangle$ calculated using the effective action for the stalk range (6.8).
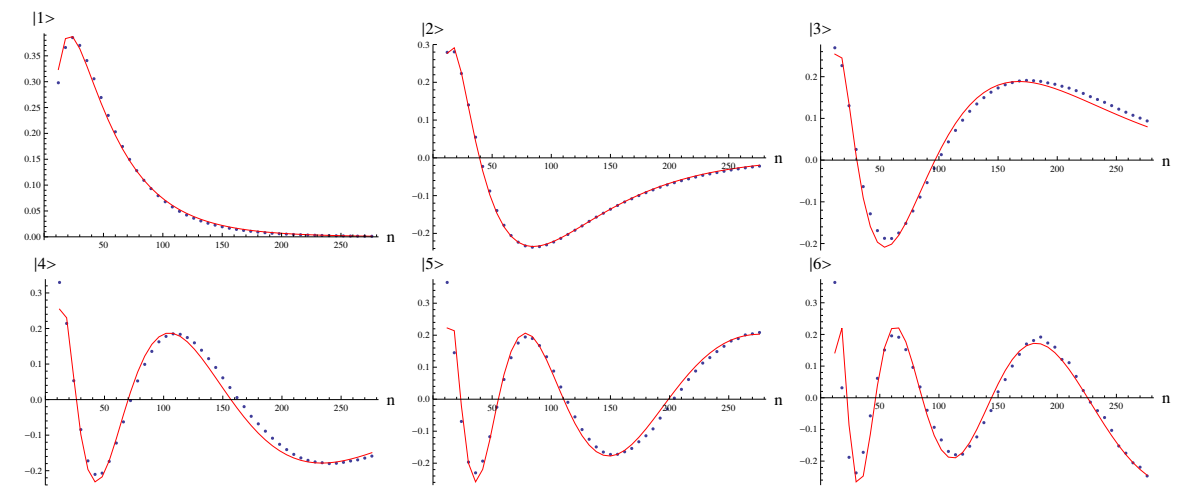

Figure 17. The first six eigenvectors of the measured $\hat{M}$ (blue dots) and "theoretical" matrix $\hat{M}^{(t h)}$ (red line). $\hat{M}^{(t h)}$ was calculated using the effective action for the stalk range (6.8).

Summing up:

$$
S_{\mathrm{eff}}^{\text {stalk }}=\sum_{t} \frac{1}{\Gamma}\left[\frac{\left(n_{t}-n_{t+1}\right)^{2}}{n_{t}+n_{t+1}-2 n_{0}}+\mu\left(\frac{n_{t}+n_{t+1}}{2}\right)^{1 / 3}-\lambda \frac{n_{t}+n_{t+1}}{2}+\delta\left(\frac{n_{t}+n_{t+1}}{2}\right)^{-\rho}\right],
$$

where the last term is not well determined.

Using the parameters of the effective action determined by a best fit, we can calculate the "theoretical" transfer matrix $\hat{M}^{(t h)}$ using (6.1) and (6.8). To appreciate the quality of this approximation we present a table of six lowest eigenvalues of the measured $\hat{M}$ and the "theoretical" matrix $\hat{M}^{(t h)}$ (table 5), as well as figures of the comparison of their six lowest eigenvectors. In each case the continuous line corresponds to the $\hat{M}^{(t h)}$ (figure 17).

\section{Discussion and conclusions}

CDT comes with a transfer matrix $\left\langle T|\mathcal{M}| T^{\prime}\right\rangle$. The way CDT is defined allows us to measure certain distributions, say $P^{t_{\text {tot }}}\left(n_{t}\right)$ and $P^{t_{\text {tot }}}\left(n_{t}, m_{t+\Delta t}\right)$, of three-volumes $n_{t}$. These distributions have an exact definition in terms of the transfer matrix $\mathcal{M}$ and the density 
matrices $\tilde{\rho}(n)$ which are projections onto the subspace of three-dimensional combinatorial triangulations $\mathcal{T}(3)$ of $S^{3}$ spanned by the triangulations with $n$ tetrahedra. (see eqs. (2.6)(2.8)). While the transfer matrix is defined on the large vector space spanned by the elements in $\mathcal{T}(3)$, the actual data coming from Monte Carlo simulations seem to allow for a much simpler description in terms of an "effective" transfer matrix $M$, only labeled by abstract vectors $|n\rangle$ referring only to the three-volume. Not only that: basically over the whole range of $n_{t}$ the data are described by a transfer matrix which can be represented as

$$
\langle n|M| m\rangle=\mathcal{N} \mathrm{e}^{-L_{\mathrm{eff}}(n, m)},
$$

where $L_{\text {eff }}(n, m)$ is given by

$$
L_{\mathrm{eff}}(n, m)=\frac{1}{\Gamma}\left[\frac{(n-m)^{2}}{n+m-2 n_{0}}+\mu\left(\frac{n+m}{2}\right)^{1 / 3}-\lambda\left(\frac{n+m}{2}\right)+\delta\left(\frac{n+m}{2}\right)^{-\rho}\right],
$$

and with a corresponding effective action

$$
S_{\text {eff }}=\sum_{t} L_{\text {eff }}\left(n_{t}, n_{t+1}\right)
$$

The last term in (7.2) unfortunately is not very well determined. For large $n_{t}$ we can not really observe it. The first terms seem to fit that data perfectly with the present statistics. For small $n_{t}$ one can detect a deviation of the data from the expression (7.2) with only the first terms included. However, as mentioned above we could not determine $\delta$ and $\rho$ well if we added a term like $\delta\left(\frac{n+m}{2}\right)^{-\rho}$. In addition the values we obtain depend on the specific merging of the three different distributions one observes for small $n_{t}$, a somewhat ad hoc procedure. Thus we cannot claim that we have a result which is discretization independent. We are caught in an unfortunate dilemma: we want to go to small $n_{t}$ in order to observe this term, which indicates corrections to the simplest minisuperspace action. However, taking $n_{t}$ small also brings us into the region where discretization effects are likely to be important. It is possible that one can find a window where $n_{t}$ is small enough for the term to be observed via high statistic measurements, but where $n_{t}$ is large enough to avoid discretization effects, but we have not yet pursued this in a systematic way.

This discussion highlights an important advantage of the present method: since $t_{\text {tot }}$ is small, we are effectively simulating much smaller systems than in the traditionally "full" CDT computer simulations. In this way we can actually obtain measurements of high statistics with relatively moderate computer resources and in a finite amount of time.

The amazing accuracy with which the effective transfer matrix seems to be described by eq. (7.1) indicates that one obtains a good approximation to the partition function by writing:

$$
\mathcal{Z}_{t_{\text {tot }}}=\operatorname{tr} M^{t_{\text {tot }}}=\sum_{n_{t_{i}}} \mathrm{e}^{-S_{\text {eff }}\left[n_{t_{i}}\right]}
$$

where $S_{\text {eff }}\left[n_{t_{i}}\right]$ is the effective action (7.2)-(7.3). We have strictly speaking only shown that this expression is a good approximation for some special values of the bare coupling constant of the Einstein-Hilbert action $S_{R}$, given by eq. (3.1). However, without much doubt 
any choice of the bare coupling constants which places us well inside the so-called de Sitter phase will allow for a description in terms of an $S_{\text {eff }}\left[n_{t_{i}}\right]$, just with different $\Gamma, \mu$ and $\lambda$. Let us assume that this is also true in the two other phases which have been observed in the "full" CDT theory. If this is the case one can actually use $S_{\text {eff }}\left[n_{t_{i}}\right]$ and the expression (7.4) to study the phase structure of CDT. This is of course much easier than using the full system. This is precisely what has been done in a recent paper [57]. Seemingly one obtains a good qualitative description of the CDT phase diagram (and also new interesting phase structures), corroborating the conjecture that the functional form (7.2)-(7.3) might be sufficient to describe CDT for all choices of the bare coupling constants of the Einstein-Hilbert action (3.1). Checking this is an obvious task for the future. However, an even more interesting application of the multi-canonical Monte Carlo simulation method developed here is that it might allow us to investigate the CDT phase transitions in more detail. A possible UV scaling limit of the CDT theory has to be associated with these phase transitions.

\section{Acknowledgments}

One of the authors (JJ) thanks the Polish Ministry of Science Grants No. N N202 22913 and 182/N-QGG/2008/0. JA would like to thank the Institute of Theoretical Physics and the Department of Physics and Astronomy at Utrecht University for hospitality and financial support. JA also thanks the Perimeter Institute for hospitality and financial support. JA and AG thank the Danish Research Council for financial support via the grant "Quantum gravity and the role of Black holes". AG acknowledges a partial support by the Polish Ministry of Science grant N N202 229137 (2009-2012).

Open Access. This article is distributed under the terms of the Creative Commons Attribution License which permits any use, distribution and reproduction in any medium, provided the original author(s) and source are credited.

\section{References}

[1] J. Ambjørn, J. Jurkiewicz and R. Loll, Quantum gravity as sum over spacetimes, Lect. Notes Phys. 807 (2010) 59 [arXiv:0906.3947] [InSPIRE].

[2] J. Ambjørn, J. Jurkiewicz and R. Loll, The universe from scratch, Contemp. Phys. 47 (2006) 103 [hep-th/0509010] [INSPIRE].

[3] J. Ambjørn, A. Görlich, J. Jurkiewicz and R. Loll, Nonperturbative quantum gravity, arXiv:1203.3591 [INSPIRE].

[4] J. Ambjørn, J. Jurkiewicz and R. Loll, Dynamically triangulating Lorentzian quantum gravity, Nucl. Phys. B 610 (2001) 347 [hep-th/0105267] [INSPIRE].

[5] J. Ambjørn, J. Jurkiewicz and R. Loll, A nonperturbative Lorentzian path integral for gravity, Phys. Rev. Lett. 85 (2000) 924 [hep-th/0002050] [inSPIRE].

[6] P. Hořava, Quantum gravity at a Lifshitz point, Phys. Rev. D 79 (2009) 084008 [arXiv:0901.3775] [INSPIRE].

[7] P. Hořava and C.M. Melby-Thompson, General covariance in quantum gravity at a Lifshitz point, Phys. Rev. D 82 (2010) 064027 [arXiv: 1007.2410] [INSPIRE]. 
[8] P. Hořava and C.M. Melby-Thompson, Anisotropic conformal infinity, Gen. Rel. Grav. 43 (2011) 1391 [arXiv: 0909.3841] [INSPIRE].

[9] P. Hořava, General covariance in gravity at a Lifshitz point, Class. Quant. Grav. 28 (2011) 114012 [arXiv:1101.1081] [INSPIRE].

[10] S. Weinberg, Ultraviolet divergences in quantum theories of gravitation, in General relativity: Einstein centenary survey, S.W. Hawking and W. Israel eds., Cambridge University Press, Cambridge U.K. (1979), pg. 790.

[11] H. Kawai and M. Ninomiya, Renormalization group and quantum gravity, Nucl. Phys. B 336 (1990) 115 [INSPIRE].

[12] H. Kawai, Y. Kitazawa and M. Ninomiya, Scaling exponents in quantum gravity near two-dimensions, Nucl. Phys. B 393 (1993) 280 [hep-th/9206081] [INSPIRE].

[13] H. Kawai, Y. Kitazawa and M. Ninomiya, Ultraviolet stable fixed point and scaling relations in $(2+\epsilon)$-dimensional quantum gravity, Nucl. Phys. B 404 (1993) 684 [hep-th/9303123] [INSPIRE].

[14] H. Kawai, Y. Kitazawa and M. Ninomiya, Renormalizability of quantum gravity near two-dimensions, Nucl. Phys. B 467 (1996) 313 [hep-th/9511217] [InSPIRE].

[15] T. Aida, Y. Kitazawa, H. Kawai and M. Ninomiya, Conformal invariance and renormalization group in quantum gravity near two-dimensions, Nucl. Phys. B 427 (1994) 158 [hep-th/9404171] [INSPIRE].

[16] M. Reuter, Nonperturbative evolution equation for quantum gravity, Phys. Rev. D 57 (1998) 971 [hep-th/9605030] [INSPIRE].

[17] A. Codello, R. Percacci and C. Rahmede, Investigating the ultraviolet properties of gravity with a Wilsonian renormalization group equation, Annals Phys. 324 (2009) 414 [arXiv:0805.2909] [INSPIRE].

[18] M. Reuter and F. Saueressig, Functional renormalization group equations, asymptotic safety and quantum Einstein gravity, arXiv:0708.1317 [INSPIRE].

[19] M. Niedermaier and M. Reuter, The asymptotic safety scenario in quantum gravity, Living Rev. Rel. 9 (2006) 5 [INSPIRE].

[20] H.W. Hamber and R.M. Williams, Nonlocal effective gravitational field equations and the running of Newton's G, Phys. Rev. D 72 (2005) 044026 [hep-th/0507017] [INSPIRE].

[21] D.F. Litim, Fixed points of quantum gravity, Phys. Rev. Lett. 92 (2004) 201301 [hep-th/0312114] [INSPIRE].

[22] M. Niedermaier, The asymptotic safety scenario in quantum gravity: an introduction, Class. Quant. Grav. 24 (2007) R171 [gr-qc/0610018] [INSPIRE].

[23] T. Regge, General relativity without coordinates, Nuovo Cim. 19 (1961) 558 [INSPIRE].

[24] F. David, Planar diagrams, two-dimensional lattice gravity and surface models, Nucl. Phys. B 257 (1985) 45 [inSPIRE].

[25] V. Kazakov, A.A. Migdal and I. Kostov, Critical properties of randomly triangulated planar random surfaces, Phys. Lett. B 157 (1985) 295 [INSPIRE].

[26] J. Ambjørn, B. Durhuus and J. Fröhlich, Diseases of triangulated random surface models and possible cures, Nucl. Phys. B 257 (1985) 433 [INSPIRE]. 
[27] D. Boulatov, V. Kazakov, I. Kostov and A.A. Migdal, Analytical and numerical study of the model of dynamically triangulated random surfaces, Nucl. Phys. B 275 (1986) 641 [INSPIRE].

[28] J. Ambjørn, B. Durhuus, J. Fröhlich and P. Orland, The appearance of critical dimensions in regulated string theories, Nucl. Phys. B 270 (1986) 457 [INSPIRE].

[29] A. Billoire and F. David, Microcanonical simulations of randomly triangulated planar random surfaces, Phys. Lett. B 168 (1986) 279 [INSPIRE].

[30] V. Knizhnik, A.M. Polyakov and A. Zamolodchikov, Fractal structure of $2 D$ quantum gravity, Mod. Phys. Lett. A 3 (1988) 819 [InSPIRE].

[31] F. David, Conformal field theories coupled to $2 D$ gravity in the conformal gauge, Mod. Phys. Lett. A 3 (1988) 1651 [INSPIRE].

[32] J. Distler and H. Kawai, Conformal field theory and $2 D$ quantum gravity or who's afraid of Joseph Liouville?, Nucl. Phys. B 321 (1989) 509 [INSPIRE].

[33] P.H. Ginsparg and G.W. Moore, Lectures on $2 D$ gravity and $2 D$ string theory, in Proceedings, Recent directions in particle theory, Boulder U.S.A. (1992), pg. 277 [YCTP-P23-92] [LA-UR-92-3479] [hep-th/9304011] [INSPIRE].

[34] P. Di Francesco, P.H. Ginsparg and J. Zinn-Justin, $2 D$ gravity and random matrices, Phys. Rept. 254 (1995) 1 [hep-th/9306153] [INSPIRE].

[35] J. Ambjørn and S. Varsted, Entropy estimate in three-dimensional simplicial quantum gravity, Phys. Lett. B 266 (1991) 285 [INSPIRE].

[36] M. Agishtein and A.A. Migdal, Three-dimensional quantum gravity as dynamical triangulation, Mod. Phys. Lett. A 6 (1991) 1863 [Erratum ibid. A 6 (1991) 2555] [INSPIRE].

[37] D. Boulatov and A. Krzywicki, On the phase diagram of three-dimensional simplicial quantum gravity, Mod. Phys. Lett. A 6 (1991) 3005 [InSPIRE].

[38] J. Ambjørn, D. Boulatov, A. Krzywicki and S. Varsted, The vacuum in three-dimensional simplicial quantum gravity, Phys. Lett. B 276 (1992) 432 [INSPIRE].

[39] J. Ambjørn and S. Varsted, Three-dimensional simplicial quantum gravity, Nucl. Phys. B 373 (1992) 557 [INSPIRE].

[40] J. Ambjørn and J. Jurkiewicz, Four-dimensional simplicial quantum gravity, Phys. Lett. B 278 (1992) 42 [INSPIRE].

[41] M. Agishtein and A.A. Migdal, Simulations of four-dimensional simplicial quantum gravity, Mod. Phys. Lett. A 7 (1992) 1039 [inSPIRE].

[42] P. Bialas, Z. Burda, A. Krzywicki and B. Petersson, Focusing on the fixed point of $4 D$ simplicial gravity, Nucl. Phys. B 472 (1996) 293 [hep-lat/9601024] [INSPIRE].

[43] B.V. de Bakker, Further evidence that the transition of $4 D$ dynamical triangulation is first order, Phys. Lett. B 389 (1996) 238 [hep-lat/9603024] [INSPIRE].

[44] S. Catterall, R. Renken and J.B. Kogut, Singular structure in $4 D$ simplicial gravity, Phys. Lett. B 416 (1998) 274 [hep-lat/9709007] [INSPIRE].

[45] J. Ambjørn and R. Loll, Nonperturbative Lorentzian quantum gravity, causality and topology change, Nucl. Phys. B 536 (1998) 407 [hep-th/9805108] [INSPIRE].

[46] J. Ambjørn, J. Correia, C. Kristjansen and R. Loll, On the relation between Euclidean and Lorentzian 2D quantum gravity, Phys. Lett. B 475 (2000) 24 [hep-th/9912267] [InSPIRE]. 
[47] J. Ambjørn, J. Jurkiewicz and R. Loll, Emergence of a $4 D$ world from causal quantum gravity, Phys. Rev. Lett. 93 (2004) 131301 [hep-th/0404156] [INSPIRE].

[48] J. Ambjørn, J. Jurkiewicz and R. Loll, Reconstructing the universe, Phys. Rev. D 72 (2005) 064014 [hep-th/0505154] [INSPIRE].

[49] J. Ambjørn, A. Görlich, J. Jurkiewicz and R. Loll, Planckian birth of the quantum de Sitter universe, Phys. Rev. Lett. 100 (2008) 091304 [arXiv:0712.2485] [INSPIRE].

[50] J. Ambjørn, A. Görlich, J. Jurkiewicz and R. Loll, The nonperturbative quantum de Sitter universe, Phys. Rev. D 78 (2008) 063544 [arXiv:0807.4481] [INSPIRE].

[51] J. Ambjørn, A. Görlich, S. Jordan, J. Jurkiewicz and R. Loll, CDT meets Hořava-Lifshitz gravity, Phys. Lett. B 690 (2010) 413 [arXiv:1002.3298] [InSPIRE].

[52] J. Ambjørn, A. Görlich, J. Jurkiewicz and R. Loll, Geometry of the quantum universe, Phys. Lett. B 690 (2010) 420 [arXiv: 1001.4581] [INSPIRE].

[53] J. Ambjørn, S. Jordan, J. Jurkiewicz and R. Loll, A second-order phase transition in CDT, Phys. Rev. Lett. 107 (2011) 211303 [arXiv:1108.3932] [INSPIRE].

[54] J. Ambjørn, J. Jurkiewicz and R. Loll, Semiclassical universe from first principles, Phys. Lett. B 607 (2005) 205 [hep-th/0411152] [INSPIRE].

[55] J. Ambjørn et al., The semiclassical limit of causal dynamical triangulations, Nucl. Phys. B 849 (2011) 144 [arXiv:1102.3929] [INSPIRE].

[56] B.A. Berg, Introduction to multicanonical Monte Carlo simulations, Fields Inst. Commun. 26 (2000) 1 [cond-mat/9909236] [INSPIRE].

[57] L. Bogacz, Z. Burda and B. Waclaw, Quantum widening of CDT universe, arXiv:1204.1356 [INSPIRE].

[58] J. Laiho and D. Coumbe, Evidence for asymptotic safety from lattice quantum gravity, Phys. Rev. Lett. 107 (2011) 161301 [arXiv:1104.5505] [INSPIRE]. 